\title{
Realistic heterointerface model for excitonic states in growth-interrupted quantum wells
}

\author{
Vincenzo Savona \\ Institut de Théorie des Phénomènes Physiques, Ecole Polytechnique \\ Fédérale de Lausanne (EPFL), CH-1015 Lausanne, Switzerland \\ Wolfgang Langbein \\ Department of Physics and Astronomy, Cardiff University, Cardiff CF24 3 YB, United Kingdom
}

(Dated: May 21, 2018)

\begin{abstract}
We present a model for the disorder of the heterointerfaces in GaAs quantum wells including long-range components like monolayer island formation induced by the surface diffusion during the epitaxial growth process. Taking into account both interfaces, a disorder potential for the exciton motion in the quantum well plane is derived. The excitonic optical properties are calculated using either a time-propagation of the excitonic polarization with a phenomenological dephasing, or a full exciton eigenstate model including microscopic radiative decay and phonon scattering rates. While the results of the two methods are generally similar, the eigenstate model does predict a distribution of dephasing rates and a somewhat modified spectral response. Comparing the results with measured absorption and resonant Rayleigh scattering in GaAs/AlAs quantum wells subjected to growth interrupts, their specific disorder parameters like correlation lengths and interface flatness are determined. We find that the long-range disorder in the two heterointerfaces is highly correlated, having rather similar average in-plane correlation lengths of about 60 and $90 \mathrm{~nm}$. The distribution of dephasing rates observed in the experiment is in agreement with the results of the eigenstate model. Finally, we simulate highly spatially resolved optical experiments resolving individual exciton states in the deduced interface structure.
\end{abstract}

\section{INTRODUCTION}

The interfaces of a semiconductor quantum well (QW) always present some amount of disorder as a natural consequence of the physics of the growth process. This disorder determines to a large extent the excitonic optical response both in frequency and time domain. Optical spectroscopy on QW excitons is therefore able to probe the interface disorder. However, the link between the optical response, particularly resonant Rayleigh scattering (RRS), and the details of the interface structure, is not straightforward and calls for a microscopic quantum model. QW interface fluctuations affect both the potential and the kinetic contributions to the total exciton center-of-mass (COM) energy, resulting in a Schrödinger equation for the exciton COM wavefunction along the QW plane, including a static disorder potential that is related to the actual interface structure. The exciton COM wavefunction is proportional to the exciton macroscopic interband polarization and relates therefore directly to the linear optical response of the system. The eigenstates in this model present both a spatial localization within the QW plane and an inhomogeneous distribution of eigen-energies. When QWs are grown by molecular beam epitaxy, interface disorder is mostly determined by surface diffusion and segregation. When using semiconductor alloys for the well or the barrier, also alloy disorder becomes important. While surface diffusion tends to create laterally extended regions with a welldefined monolayer (ML) thickness, segregation and alloy disorder result in nanoroughness on an atomic length scale, smaller than the exciton Bohr radius $\frac{1}{\underline{1}}$. The in- terruption of the epitaxial growth at the QW interfaces leads to a longer surface diffusion length, creating ML islands in the $100 \mathrm{~nm}$ size range that have been observed in various experimental studies 2.3.4. When ML islands are sufficiently ${ }^{5}$ large compared to the excitonic Bohr radius, the exciton spectrum can split into several peaks of reduced inhomogeneous broadening ${ }^{6}$. In a simple picture these peaks are resulting from exciton states present within flat regions of different integer ML thickness, while the inhomogeneous broadening results from a residual nanoroughness. To create from this picture a realistic description of the ensemble properties of the exciton in the QW, one needs to consider the following issues: (i) the statistical distribution of size and shape of regions with different ML thickness of the interfaces; (ii) the difference of the structural parameters and the statistical correlation of the two interfaces; (iii) the lateral quantum confinement energy of the exciton states; (iv) the coupling of the exciton states to light. Once the statistical properties of the interface structure are described by a model with a small number of parameters, these parameters can be deduced from the measured RRS spectrum and dynamics.

Existing theoretical models of RRS in QWs were often based on very simplified assumptions for the shape of the disorder potential. The simplest picture, introduced in the original work on RRS theory by Savona and Zimmermann ${ }^{7}$ consisted in assuming a random potential with Gaussian or exponential correlation length in space. Although this approach can explain the principial properties of $\mathrm{RRS}, \underline{\underline{\underline{8}}}$ it is unable to predict the dynamics of more complex situations like the one considered 
here. In a previous work ${ }^{9}$, we had developed a rudimentary extension of the disorder model including a shortand a long-range disorder component, for the modeling of RRS in samples where one of the two heterointerfaces was growth interrupted.

In this work we apply the microscopic theory of the RRS optical response of excitons ${ }^{7}$ to a situation where a pronounced ML splitting is present. To this purpose, we develop a comprehensive model of the disorder potential based on a separate description of the direct and inverse heterointerfaces of the QW. The model accounts for a ML island structure at both QW interfaces, with different correlation lengths, anisotropy factors and statistical distributions of the integer values of the ML thickness. It also includes nanoroughness and a parameter that expresses the statistical correlation between the two interfaces. The experimental RRS data, both spectrally and time-resolved, were taken on a GaAs/AlAs QW sample grown with long growth interruptions at both interfaces. The amount of deposited GaAs is varying slowly across the lateral position on the sample, so that a fine control of the average QW thickness by a small fraction of one ML is achieved. This in turn produces a continuous evolution of the ML-split exciton spectrum, which replicates itself after a thickness change of an integer ML with only an energy shift given by the corresponding difference in QW confinement energy. Correspondingly, also the timeresolved RRS shows a peculiar dependence on the fractional ML thickness. We use this rich phenomenology to determine the parameters of a detailed interface model that we develop. The calculated RRS response is confronted with the measured spectral and time-dependent RRS signatures, and the effects of the various interface properties are elucidated. This analysis allows an estimation of the actual parameters characterizing the investigated sample - to a much larger extent than in previous studies based on the same RRS theory. Specifically we find an almost perfect correlation between the direct and inverse QW interfaces - a novel result that is of general relevance for epitaxial QW samples. In the second part of the work, we turn to the numerical computation of the actual exciton COM eigenstates as resulting from the disorder model. This part of our study is required in order to account for state-dependent radiative recombination and phonon scattering rates, which prove important especially in the RRS dynamics. It provides us with further insights into the microscopic structure of the single exciton states and their optical properties.

The paper is organized as follows. In Section II we describe the time-propagation RRS theory and the interface disorder model, followed by a study of the dependence of the simulated RRS on the model parameters. Section III describes the experimental data and the features of the QW interfaces, as deduced from the comparison to the model calculations. Section IV is devoted to a microscopic simulation of the exciton eigenstates and of their radiative and phonon scattering rates. Results for ensemble averages and individual disorder realizations are presented. Section V contains the conclusions and outlook of the work.

\section{THEORETICAL DESCRIPTION}

The present model is based on the theory of RRS within the exciton COM approximation, as derived by Savona and Zimmermann ${ }^{7}$. The exciton interband polarization $P$ is proportional to the exciton $\mathrm{COM}$ wavefunction along the QW plane and therefore evolves according to the time-dependent Schrödinger equation

$$
\begin{aligned}
-i \hbar \frac{\partial P(\mathbf{R}, t)}{\partial t} & =\left(-\frac{\hbar^{2}}{2 M} \nabla^{2}+V(\mathbf{R})\right) P(\mathbf{R}, t) \\
& +\mu E_{\mathrm{in}}(\mathbf{R}, t),
\end{aligned}
$$

where $\mathbf{R}=(x, y)$ is the position in the $\mathrm{QW}$ plane, $M$ is the in-plane exciton mass, $\mu$ is the dipole matrix element of the excitonic transition, and $E_{\mathrm{in}}(\mathbf{R}, t)$ is the resonant excitation field. In the present model, we neglect the vector character of the electric field and of the interband polarization. Rayleigh scattering is expected to conserve the orientation of the polarization vector of the scattered field, whereas the long-range exchange part of the Coulomb interaction can induce a rotation of this vector on a timescale of several tens of picoseconds ${ }^{10.11}$. The static COM potential $V(\mathbf{R})$ is an effective quantity, related to the actual exciton energy fluctuations via a convolution with the 1 s-exciton relative wavefunction 11 . Differently from the usual definition, we have incorporated here the average $1 s$ exciton energy $E_{\mathrm{X}}$ into $V(\mathbf{R})$. We detect the RRS in the far field and neglect time delays due to light-propagation. In linear response theory, the RRS amplitude $E_{\text {out }}(\mathbf{k}, t)$ is proportional to the Green's function $G\left(\mathbf{k}, \mathbf{k}_{\mathrm{in}}, t\right)$ of the Schrödinger equation (10), expressed in momentum space $\stackrel{7.11}{.}$ where $\mathbf{k}_{\text {in }}$ is the momentum of the incident electromagnetic field $E_{\mathrm{in}}\left(\mathbf{k}_{\mathrm{in}}, t\right)$, assumed to be a plane wave. To determine the spectrally resolved RRS $E_{\text {out }}(\mathbf{k}, \omega)=\int_{0}^{\infty} E_{\text {out }}(\mathbf{k}, t) e^{(i \omega-\gamma) t} d t$, we introduce a phenomenological polarization decay rate $\gamma$. The resulting RRS intensity

$$
I_{\mathrm{RRS}}(\mathbf{k}, t) \propto\left|E_{\text {out }}(\mathbf{k}, t)\right|^{2}, \quad I_{\mathrm{RRS}}(\mathbf{k}, \omega) \propto\left|E_{\text {out }}(\mathbf{k}, \omega)\right|^{2}
$$

is averaged over a large statistical ensemble of disorder realizations. In a similar way, we can define the exciton COM spectral function $A(\mathbf{k}, \omega)=-2 \operatorname{Im}\{G(\mathbf{k}, \mathbf{k}, \omega)\}$ as proportional to the imaginary part of the COM Green's function at equal momentum arguments. The spectral function at momentum $\mathbf{k}_{\text {in }}$, averaged over disorder realizations, is proportional to the exciton absorption spectrum (the same average is identically zero if the Green's function is taken with two differing momentum arguments). The simulated spectral function will be compared, in this and in the next Section, to the measured excitonic absorption. Throughout the paper, when quoting values for frequencies or rates in energy units, they are multiplied by $\hbar$. 


\section{A. Model for the interface structure}

In order to model the exciton COM potential in presence of ML islands in the heterointerfaces, as in the QWs studied here, the following procedure is used. First, a random white-noise function $w_{\alpha}(\mathbf{R})$ is generated, such that $\left\langle w_{\alpha}(\mathbf{R}) w_{\alpha}\left(\mathbf{R}^{\prime}\right)\right\rangle=\delta\left(\mathbf{R}-\mathbf{R}^{\prime}\right)$. The index $\alpha=1,2$ denotes the two $\mathrm{QW}$ heterointerfaces. We then derive a spatially correlated function of unitary standard deviation and zero mean value

$W_{\alpha}(\mathbf{R}) \propto \int d \mathbf{R}^{\prime} w_{\alpha}\left(\mathbf{R}^{\prime}\right) \exp \left(-\frac{\left(x-x^{\prime}\right)^{2}}{2 \xi_{x, \alpha}^{2}}-\frac{\left(y-y^{\prime}\right)^{2}}{2 \xi_{y, \alpha}^{2}}\right)$

Here we have introduced the Gaussian correlation lengths $\xi_{j, \alpha}$, with $j=x, y$, which will determine the length scale of the ML islands along the $x$ and $y$ directions, respectively. The separate length scales for the two directions allow to describe a spatial anisotropy of the ML structure. We assume equal orientation of the anisotropy axis for both interfaces since we expect the microscopic origin of the anisotropy to be given by the anisotropic reconstruction of the [001] growth surface along the [110] in-plane direction, equal for the whole epitaxially grown structure. We express the two correlation lengths as a function of an anisotropy parameter $\epsilon_{\alpha}$ and of an isotropic correlation length $\xi_{\alpha}$, defining $\xi_{x, \alpha}=\epsilon_{\alpha}^{1 / 2} \xi_{\alpha}$ and $\xi_{y, \alpha}=\epsilon_{\alpha}^{-1 / 2} \xi_{\alpha}$. We proceed by generating a ML step function from $W_{\alpha}$ that models the property of a growth surface to form compact regions of integer ML coverage due to the lateral binding energy ${ }^{12}$ of the adatoms, as opposed to a random arrangement. We use the function

$$
I_{\alpha}(\mathbf{R})=\text { Round }\left[\frac{W_{\alpha}(\mathbf{R})}{\zeta_{\alpha}}+\Delta^{\prime}+\Delta \cdot \delta_{\alpha, 2}\right] \text {, }
$$

where the function Round $[x]$ denotes the nearest integer of a real number $x$. The quantity $\zeta_{\alpha}$ is the interface flatness, controlling the height variance of the interface to be approximately $\zeta_{\alpha}^{-1}$ MLs. The parameter $\Delta^{\prime} \in[0,1]$ is a fractional ML shift that describes the fractional ML coverage of the first interface, i.e. the AlAs surface on which the GaAs QW is grown. The spatial average of the ML coverage $\left\langle I_{1}\right\rangle_{\mathbf{R}}$ is approximately $\Delta^{\prime}$ for interfaces that consist of several ML steps, i.e. $\zeta \lesssim 3$. This is the case for the simulations presented later on. For flatter interfaces the fractional ML coverage is a non-linear function of $\Delta^{\prime}$ (see upper inset of Fig.11). The same arguments hold for $\left\langle I_{2}\right\rangle_{\mathbf{R}}$ and $\Delta^{\prime}+\Delta$. Due to an unavoidable slight misorientation $(>1$ ") of the substrate with respect to the [001] direction, the fractional ML coverage of the interface is expected to vary over many ML thicknesses across the excited surface area, which has a lateral extension of about $10^{6} \mathrm{ML}(300 \mu \mathrm{m})$. We will therefore use a uniform distribution of $\Delta^{\prime}$ over $[0,1]$ in the statistical ensembles for the simulations. The quantity $\Delta$ is shifting the second heterointerface with respect to the first one, as specified by the Kronecker $\delta_{\alpha, 2}$ in Eq. (4). It defines the average distance between the first and the second heterointerface, i.e. the average QW thickness. This thickness can be accurately varied in the experiment by displacing the laser spot along the QW plane since the average QW thickness is given by the deposited amount of GaAs within the surface diffusion length and varies only by about $0.1 \mathrm{ML}$ over $300 \mu \mathrm{m}$ lateral extension ${ }^{13}$. In this argument, we assume that $\mathrm{Ga}$ and $\mathrm{Al}$ atoms do not evaporate from the surface once deposited. This assumption is justified for the growth parameters used for the investigated samples.

We continue by deriving a local exciton energy $U(\mathbf{R})=F\left(I_{2}(\mathbf{R})-I_{1}(\mathbf{R})\right)$ from the local ML-thickness $I_{2}-I_{1}$. The dependence of the exciton energy on the MLthickness has the form

$$
F(\Delta)=a_{0}+a_{1} / \Delta+a_{2} / \Delta^{2}+a_{3} / \Delta^{3}+a_{4} / \Delta^{4}
$$

where the parameters $a_{n}$ are fitted to the experimental data 13 obtained on the same sample by measuring the exciton peak position at equivalent fractional ML thicknesses over a large range of thicknesses (see Fig.11). A random, spatially uncorrelated potential $u(\mathbf{R})$ is summed in order to account for short-range disorder originating from segregation. The COM potential $V(\mathbf{R})$ is obtained by convoluting $U(\mathbf{R})+u(\mathbf{R})$ with the electron and hole probability distribution in the 1 s-exciton 52 $\left|\phi\left(\mathbf{R}^{\prime}\right)\right|^{2} \propto \exp \left(-\left|\mathbf{R}-\mathbf{R}^{\prime}\right| / a_{\mathrm{B}}\right)$, using an exciton Bohr radius $a_{\mathrm{B}}=8 \mathrm{~nm}$. This value was deduced from the measured diamagnetic shift $\alpha_{d}=33 \mu \mathrm{eV} / \mathrm{K}$ and the reduced mass $\mu^{*}=0.0586 m_{0}$ for a $37 \mathrm{ML}$ thick GaAs/AlAs quantum well 14 using the formula $\alpha_{d}=a_{\mathrm{B}}^{2}\left(3 e^{2}\right) /\left(16 \mu^{*}\right)$ valid for an in-plane exciton wavefunction proportional to $\exp \left(-r / a_{\mathrm{B}}\right)$, with the in-plane electron-hole distance $r$. The amplitude of the short-range disorder $u(\mathbf{R})$ is parameterized by $\sigma$, expressing its standard deviation after the convolution with the 1 s-exciton relative wavefunction.

Within this model, particular attention must be payed to the statistical correlation between the ML fluctuations of the two interfaces. In the epitaxial growth, a substantial amount of correlation can be expected between the ML fluctuations of the lower and upper interfaces 15 . We introduce this correlation into our model via the two white-noise functions $w_{\alpha}(\mathbf{R})$ that enter Eq. (3). The limiting case of fully correlated heterointerfaces is obtained by taking $w_{1}=w_{2}$. Conversely, the fully uncorrelated case results from using two uncorrelated white noise functions $w_{1}$ and $w_{2}$. In order to model the intermediate case of partial correlation, we introduce two mutually uncorrelated white-noise functions $w(\mathbf{R})$ and $w^{\prime}(\mathbf{R})$. We could then proceed by simply defining $w_{1}=w$ and $w_{2}=\kappa w+\left(1-\kappa^{2}\right)^{1 / 2} w^{\prime}$, therefore assuming a uniform correlation $\kappa$ for all the Fourier components of $w_{1,2}$. However, the total number of Ga atoms deposited over length scales larger than the surface diffusion length has to be conserved in the growth process. Even though the atoms are deposited at random in molecular beam epitaxy, the total number of Ga atoms contained in the QW within an area given by the surface diffusion length is $>10^{6}$, so that the statistical error in the QW thickness is be- 


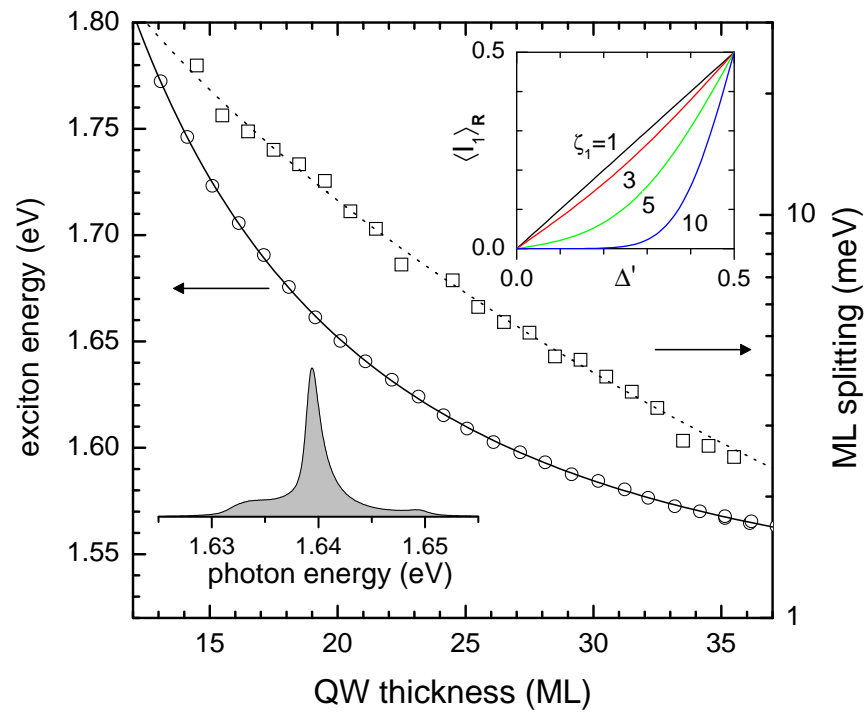

FIG. 1: Measured exciton transition energy (circles) versus nominal QW thickness $\Delta$, and the fitted $F(\Delta)$ (solid line) using $\left(a_{0}=1.516, a_{1}=-0.233, a_{2}=92.5, a 3=-806, a_{4}=\right.$ 2739) eV. The measured exciton PL peak energy at a thickness resulting in a dominating single ML peak (see lower inset) is attributed to the exciton potential $F(\Delta)$ at +0.15 fractional ML thickness. This slight shift is needed to account for the quantum confinement due to short-range disorder, which is shifting the peak in the optical spectrum to slightly lower energy with respect to the nominal zero-point (spatial average) of the disorder potential (see e.g. Fig. 5 in Ref. 11). The value $+0.15 \mathrm{ML}$ is giving the best fit between RRS measurements and simulations at $\Delta \approx 38$. Additionally, the measured ML splitting (squares) and its fit $d F / d \Delta$ (dashed line) is shown. The upper inset shows the average ML coverage of the first interface $\left\langle I_{1}\right\rangle_{\mathbf{R}}$ as function of $\Delta^{\prime}$.

low $10^{-3}$, a fluctuation that can be neglected compared to the interface roughness. The long-wavelength components of the ML fluctuations in the two interfaces have therefore to be fully correlated. In order to accommodate this requirement, we modify the above approach for the long-wavelength component of $w_{2}$. Using the Fourier transforms $w_{\mathbf{k}}$ and $w_{\mathbf{k}}^{\prime}$ of the two generating functions, we define the function $w_{2}(\mathbf{R})$ as the inverse Fourier transform of

$$
w_{2, \mathbf{k}}=f_{k} w_{\mathbf{k}}+\left(1-f_{k}^{2}\right)^{1 / 2} w_{\mathbf{k}}^{\prime}
$$

with

$$
f_{k}=(1-\kappa) \exp \left(-\frac{k^{2}}{l_{c}^{2}}\right)+\kappa,
$$

where $l_{c}$ is a surface diffusion length. In this way, the parameter $\kappa \in[0,1]$ still represents the correlation between the two interfaces for the Fourier components having wavelengths shorter than $l_{c}$, while for longer wavelengths the interfaces are fully correlated as dictated by the conservation of $\mathrm{Ga}$ atoms. It the following simulations, $l_{c}$ is always much larger than the correlation lengths $\xi_{\alpha}$, and has therefore no significant effect. We used $l_{c}=500 \mathrm{~nm}$.

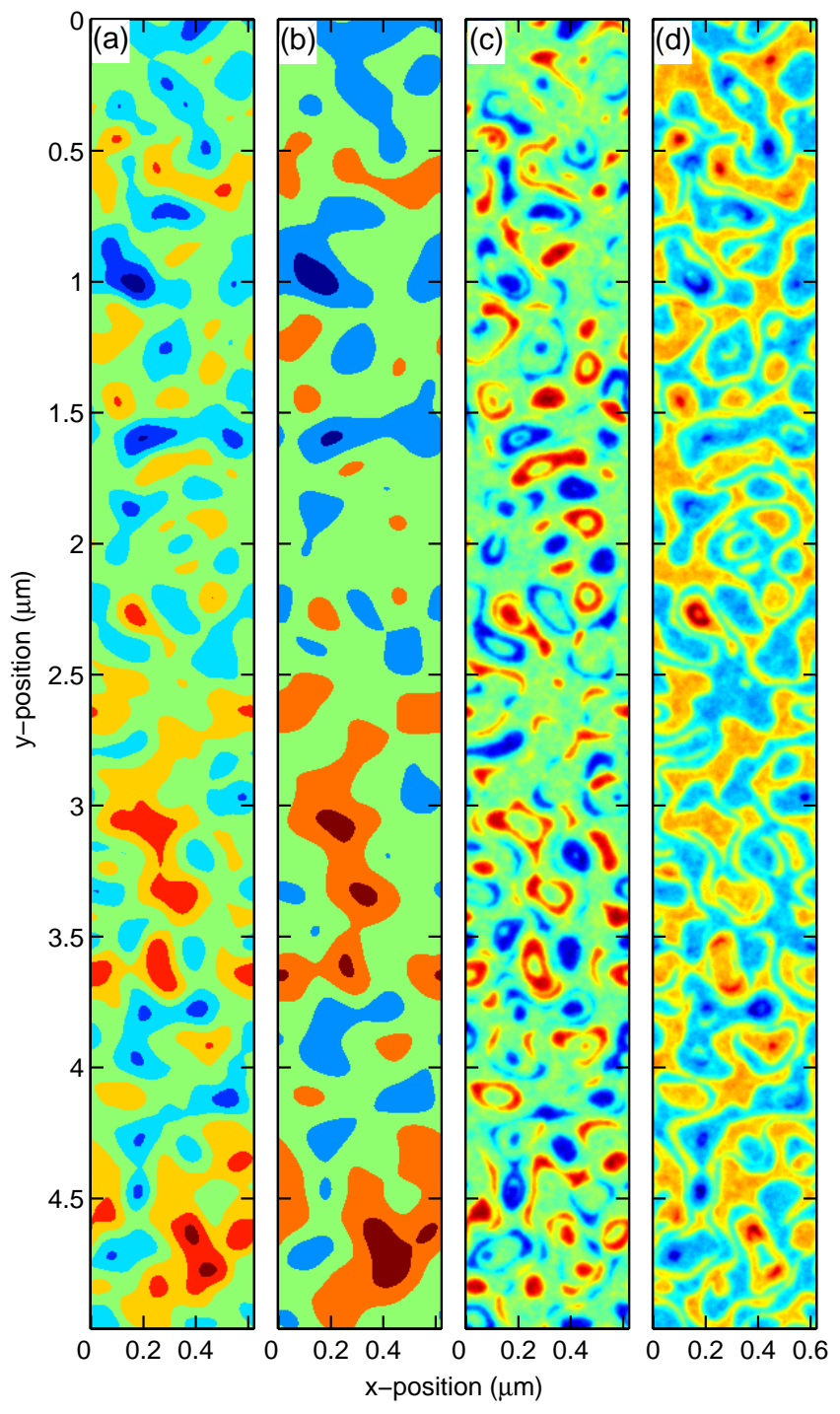

FIG. 2: (a) Color plot of the first interface profile $I_{1}(\mathbf{R})$ for $\Delta=38.0$ while $\Delta^{\prime}$ is varied in the interval $[0,1]$ along the y-position. The color steps represent ML height steps. (b) Same for the second interface profile $I_{2}(\mathbf{R})$. (c) COM potential $V(\mathbf{R})$ for an integer monolayer thickness $\Delta=38.0$. (d) Same for half-integer monolayer thickness $\Delta=38.5$. The parameters for this potential are given in Table】 and the correlation is $\kappa=1$. The color scale in c) \& d) covers $5 \mathrm{meV}$.

The conservation of $\mathrm{Ga}$ atoms also requires that the long wavelength components of $W_{1,2}$ created from $w_{1,2}$ are equal, which relates the correlation length and the flatness parameters of both interfaces by $\xi_{1} / \zeta_{1}=\xi_{2} / \zeta_{2}$. We will assume this relation to hold throughout this work.

\section{B. Simulations}

The time-dependent RRS field is simulated by solving the time-dependent Schrödinger equation with the poten- 
tial $V(\mathbf{R})$, according to Eq. (11). The Fourier transform of the interband polarization then provides the RRS amplitude in wavevector and frequency domain ${ }^{7}$. The RRS intensity computed in this way is averaged over $10^{3}-10^{4}$ realizations of $V(\mathbf{R})$ to create a statistically relevant ensemble average, converging to its asymptotic value within $3-1 \%$ accuracy. The remaining statistical fluctuations are only appreciable in the time-resolved RRS intensity at long times, at which the asymptotic RRS dynamics is very slow. It was shown ${ }^{7}$ that the realization average coincides with the speckle average which is performed in the experiment by using a large excitation region of about $(200 \mu \mathrm{m})^{2}$ and averaging over a small span of scattering directions $\underline{\underline{16}}$ This correspondence is very useful, as simulating directly a large area is numerically prohibitive. We use typically a $(500 \mathrm{~nm})^{2}$ simulation area over $64 \times 64$ grid points with periodic boundary conditions. We assume a plane-wave excitation field at $\mathbf{k}=0$ and take the scattered RRS field at the smallest available wavevector value $\mathrm{k}=4 \pi / \mu \mathrm{m}$ on the simulation grid. The average ML thickness $\Delta$ is known for each position on the sample by a previous study $\underline{13}$. The free parameters used in this model are therefore the correlation lengths $\xi_{\alpha}$, the flatness $\zeta_{1}$, the anisotropy constants $\epsilon_{\alpha}$, the correlation $\kappa$, and the short-range disorder amplitude $\sigma$.

In this Section we proceed by discussing the simulated, spectrally or time-resolved RRS intensity for the disorder parameters that best describe our experimental data, which are presented in full detail in the next Section (see Fig. (9). These parameters were obtained by a trial-and-error procedure that consisted in (i) performing a simulation with a given parameter set, (ii) comparing to the measured data (both spectral and time-resolved), and (iii) adjusting heuristically the parameters and going back to step (i). A standard best-fit procedure is made prohibitive by the long computing time required for a single simulation (a few hours for TP, a few days for ES). Starting from this parameter set, indicated in Table凹(TP) we discuss how varying the different parameters of the model affects the outcome of the simulations, also in light of previous reports concerning the interface structure in epitaxial crystal growth. This analysis also provides estimations of the relative accuracy of the parameter values determined from the comparison to the experiments.

Fig.2(a) and (b) show examples of the two interface functions $I_{1}(\mathbf{R})$ and $I_{2}(\mathbf{R})$ for the interface parameters found to reproduce the measured RRS (see Table \TP). The fractional ML shift $\Delta^{\prime}$ was varied linearly from 0

\begin{tabular}{|l|c|c|c|c|c|c|c|c|}
\hline Model & $\xi_{1}$ & $\xi_{2}$ & $\zeta_{1}$ & $\zeta_{2}$ & $\epsilon_{1}$ & $\epsilon_{2}$ & $\sigma$ & $\gamma$ \\
\hline $\mathrm{TP}$ & $65 \mathrm{~nm}$ & $87 \mathrm{~nm}$ & 1.2 & 1.6 & 1.0 & 1.0 & $0.8 \mathrm{meV}$ & $10 \mu \mathrm{eV}$ \\
$\mathrm{ES}$ & $55 \mathrm{~nm}$ & $82 \mathrm{~nm}$ & 1.46 & 2.2 & 1.0 & 1.0 & $0.8 \mathrm{meV}$ & - \\
\hline
\end{tabular}

TABLE I: Parameters of the interface model obtained by adjusting the simulations to the measured RRS data for the time-propagation (TP) and the eigenstate (ES) calculations.

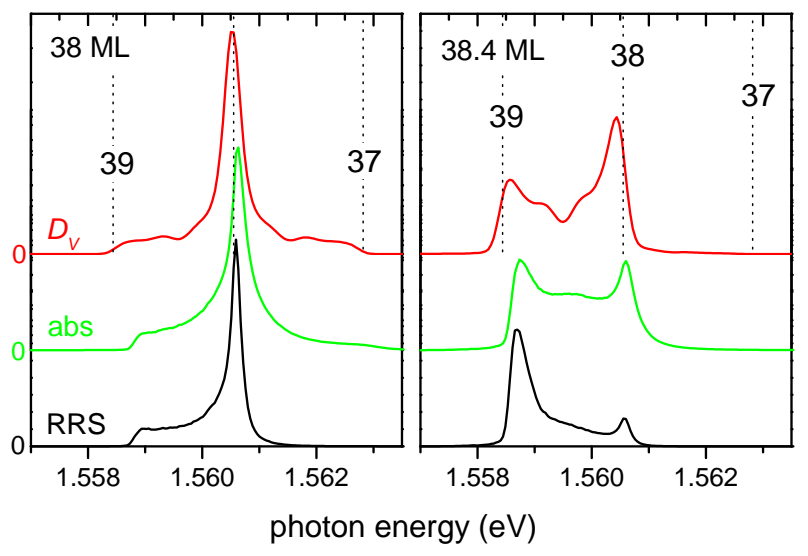

FIG. 3: Calculated spectral disorder potential density $D_{V}(\omega)$, absorption (abs), and RRS intensity (RRS) for $\Delta=$ 38.0 and 38.4 ML, other parameters as in Fig.2 Data are vertically offset for clarity. The dotted lines represent the potential $F(\Delta)$ for the labelled integer $\Delta$.

to 1 along the $y$ direction. These false color pictures illustrate how the next ML level gradually develops when moving along the $y$ direction. The two interfaces are fully correlated $(\kappa=1)$, as is reflected in the similar shapes of the two ML patterns. The non-equal correlation lengths $\xi_{1}<\xi_{2}$ result in smaller features and more irregular patterns being present on the first interface (a). The resulting COM potential $V(\mathbf{R})$ for integer and halfinteger ML thickness $\Delta$ of the QW are displayed in (c) and (d). Both pictures show that the variation of the fractional ML shift $\Delta^{\prime}$ is compensated when subtracting the ML profiles (Eq.4) of the two interfaces, and does thus not significantly affect the COM potential. On the other hand, the difference between integer and halfinteger ML thickness appears clearly in the plots. Because of interface correlation, for integer ML thickness the ML steps on the two interfaces appear synchronously and, in the limiting case of identical interfaces, the QW thickness would remain constant $\underline{53}$ The fluctuations in the QW thickness are thus due to the different characteristic sizes of ML islands on the two interfaces which give rise to ring and stripe patterns in the $\mathrm{QW}$ thickness and thus in $V(\mathbf{R})$, as can be seen in Fig.2(c). In the case of half-integer ML thickness the steps on the two interfaces are out of phase, and the ML steps are likely to appear on one interface only, giving rise to flat regions in $V(\mathbf{R})$ as displayed in Fig.2(d), which are representing two rather well defined ML thicknesses.

The spectral density

$$
D_{V}(\omega)=A^{-1} \int \delta(V(\mathbf{R})-\hbar \omega) d \mathbf{R}
$$

of the resulting disorder potential is given in Fig. 3 For integer ML thickness $\Delta=38.0, D_{V}$ has a dominant peak around $F(38)$, with tails towards the high and low energy extending to the potentials of the adjacent integer ML thicknesses. Due to the averaging over the exciton 


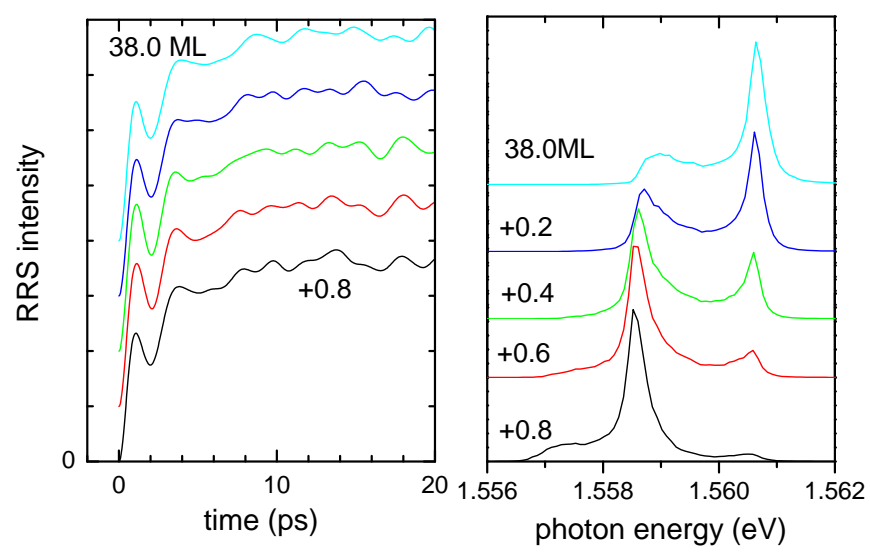

FIG. 4: Calculated time- (a) or spectrally (b) resolved RRS intensity versus the fractional ML thickness $\Delta$ for a GaAs/AlAs interface of long and a AlAs/GaAs interface of short correlation length $\left(\xi_{1}=25 \mathrm{~nm}, \xi_{2}=200 \mathrm{~nm}\right)$, and a ML flatness $\zeta_{1}=2.5$. Data are offset for clarity.

size, the regions of adjacent ML thickness do not result in such distinct peaks since they have a small spatial extension. The calculated absorption peak is shifted to higher energies due to the finite mass of the exciton giving a lateral quantization energy. Furthermore, only the lower energy ML is visible in the absorption, but not the higher one. This asymmetry relates to the small momentum of the light, which essentially probes the $k=0$ components of the wavefunctions. With increasing energy, the wavefunctions get more nodes, i.e. a smaller $k=0$ component, so that only the lower part of the potential landscape relates to optically active exciton states. The RRS spectrum shows a similar behaviour as the absorption, but in a more pronounced way. This is expected because the RRS intensity - being proportional to the squared COM Green's function - varies as the fourth power of the COM wavefunction at small momentum, as opposed to the second power dependency characterising the absorption spectrum. For about half-integer ML thickness $\Delta=38.4, D_{V}(\omega)$ is distributed between $F(38)$ and $F(39)$, as expected. The absorption and the RRS shows two peaks, with the lower energy peak dominating, again due to the dominant $k=0$ component of the lowest energy eigenstates.

\section{Long correlation length of GaAs/AlAs interface}

The parameters of Table \define fully correlated interfaces with similar correlation lengths $\xi_{1}=65 \mathrm{~nm}$ and $\xi_{2}=87 \mathrm{~nm}$. From structural investigations, it could be expected that the islands on the GaAs surface are much larger than on the AlAs surface due to the largely different surface mobility at the growth temperature of $630^{\circ} \mathrm{C}$. On GaAs surfaces after 60 s growth interruption, islands of several hundred nanometers were observed ${ }^{2.3 .4}$. Earlier investigations, however, measured the GaAs surface after
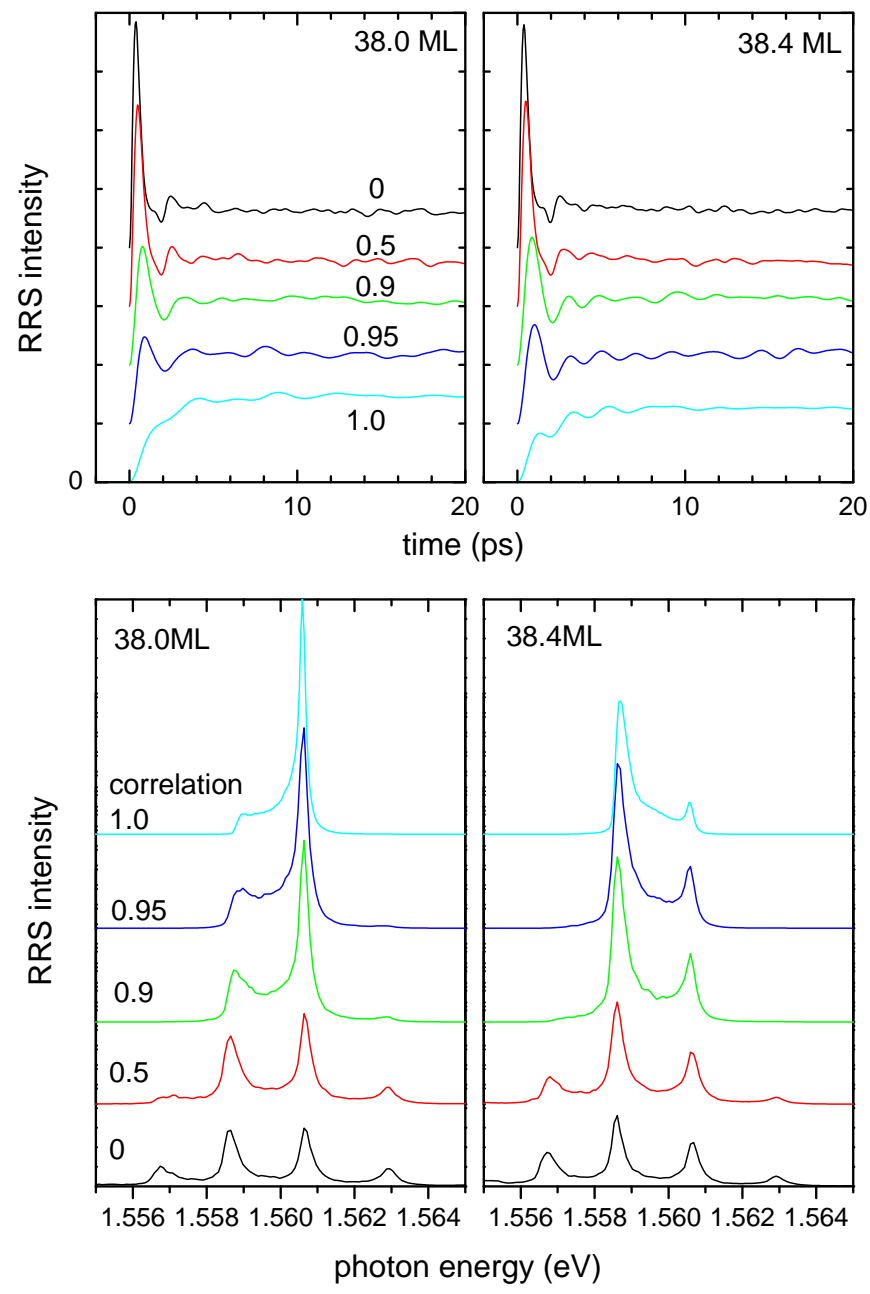

FIG. 5: Calculated time- (a) or spectrally (b) resolved RRS intensity versus the interface correlation $\kappa$. Other parameters as in table凹 Data are offset for clarity.

cooling, which can have a significantly larger correlation length than interior interfaces $\frac{17}{7}$. In order to test this expectation, we therefore discuss now the RRS spectra of excitons in interface structures with a large correlation length of the GaAs to AlAs interface of $\xi_{2}=200 \mathrm{~nm}$. This is much larger than the localization length of a ML island $\left.{ }^{5.13} \xi_{0}=\pi \hbar / \sqrt{2 M(d F / d \Delta}\right) \approx 18 \mathrm{~nm}$ for $\Delta=38$. This interface thus does not significantly influence the exciton localization. The free parameters are then the AlAs to GaAs interface correlation length $\xi_{1}$ and flatness $\zeta_{1}$. The variation of the RRS spectra with increasing $\Delta$ for 38 to 39 is best reproduced using $\xi_{1}=25 \mathrm{~nm}$ and $\zeta_{1}=2.5$, as displayed in Fig. 4. The resulting RRS dynamics, however, is essentially independent of $\Delta$, in contrast to the experimental results (see Fig. (9). This finding is insensitive to the specific values of $\xi_{1}$ and $\zeta_{1}$, and shows that two interfaces with largely different correlation lengths are not consistent with the measured RRS dynamics. 


\section{Interface Correlation}

For the case of largely different lengths $\xi_{1,2}$ of the two interfaces as discussed in the previous Subsection, the correlation $\kappa$ is not important since the ML islands of the interfaces are very different. For similar correlation lengths of the two interfaces instead, $\kappa$ is important, since for full correlation $(\kappa=1)$ only the small difference between the two correlated interfaces remains, leaving only narrow flat regions in $V(\mathbf{R})$ as seen in Fig. 2(c). Without correlation $(\kappa=0)$, on the other hand, the difference between the uncorrelated interfaces is similar to the case of a single interface with a reduced flatness. Starting from the parameters in Table \(TP), we show in Fig. 5 the effect of a decreasing correlation on the RRS spectra and dynamics. Already for a small deviation from full correlation $(\kappa=0.95)$, for which the RRS spectrum is not significantly changed, the RRS dynamics develops an initial peak indicating the presence of a disorder component of large correlation length ${ }^{7}$. This component results from the difference between the $k>1 / \xi_{2}$ Fourier components of $W_{1}$ and $W_{2}$, which does not vanish for non-perfect correlation $\kappa<1$. For even smaller values of $\kappa$, the RRS spectrum gradually develops more ML peaks $(\Delta=37,39)$, and the initial peak in the RRS dynamics gets more pronounced. The RRS dynamics becomes essentially independent of the fractional $\Delta$, a behavior inconsistent with the experiment. We thus conclude that the ML steps on the two interfaces have to be nearly perfectly correlated. This finding suggests that for our samples the surface diffusion length $l_{c}$ is actually similar to the correlation lengths $\xi_{\alpha}$, so that the almost perfect interface correlation is created by the conservation of the deposited Ga atoms. We would expect this finding to depend on the duration of the growth interruption at the GaAs to AlAs interface and the growth temperature. For long growth interruption times and increasing temperature, the surface diffusion length is increasing compared to the island size, as the surface diffusion distance is increasing with $\sqrt{t}$, while the island size is thermodynamically limited. With increasing duration of the growth interruption (GI) and growth temperature, the interface correlation is therefore expected to be diminished. This is actually supported by previous experiments. Yu et al ${ }^{18}$ have studied the PL spectra of GaAs/AlAs QWs depending on the GI time. They observe (Fig. 1 of Ref. 18) the formation of the ML splitting during the first 60 s GI, with only 2 ML peaks present, while for $120 \mathrm{~s}$ GI, already 4 peaks are visible, similar to our simulation for $\kappa=0.5$ in Fig.5. For GI GaAs QWs with mixed crystal barriers $19,20,21$ normally more than $2 \mathrm{ML}$ peaks are present. This indicates that for mixed crystal barriers, the interface disorder is partly uncorrelated, as was also observed in structural investigations ${ }^{22}$. This finding can be understood considering that a $\mathrm{Al}_{x} \mathrm{Ga}_{1-x} \mathrm{As}$ mixed crystal growth surface consists essentially only of Ga atoms that are mobile. The $\mathrm{Al}$ distribution, which determines the interface cre-
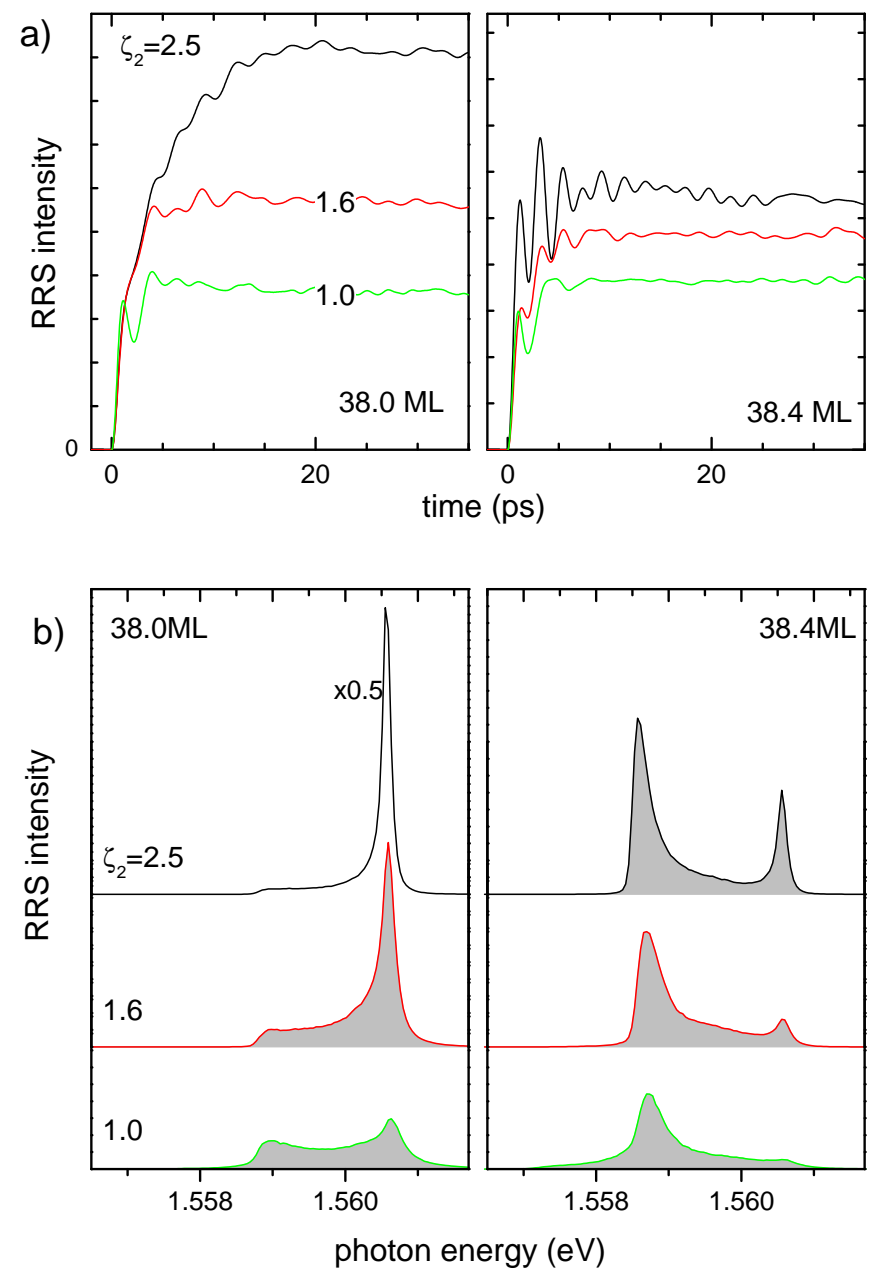

FIG. 6: Calculated time- (a) or spectrally (b) resolved RRS intensity versus the flatness $\zeta_{2}$ for $\zeta_{1} / \zeta_{2}=0.75$. Results for $\Delta=38.0$ (left) and $\Delta=38.4$ (right) are shown. Data are offset for clarity.

ated after overgrowth with GaAs, is therefore essentially unmodified during the growth interrupt. The resulting first interface is consequently dominated by short range disorder, and the ML islands formed by the growth interrupt at the second interface give rise to a larger number of ML peaks due to the missing correlated islands on the first interface.

\section{Influence of flatness and correlation lengths}

We now discuss the influence of the flatness $\zeta_{1}$ and the correlation lengths $\xi_{1,2}$ on the optical response, starting from the parameters in Table $1 \mathrm{TP})$. The correlation is taken to be unity $(\kappa=1)$, and $\zeta_{2} / \zeta_{1}=\xi_{2} / \xi_{1}$. We first change interface flatness, from the value $\zeta_{1}=1.6$, to a smoother $\zeta_{1}=2.5$, and rougher $\left(\zeta_{1}=1.0\right)$ interface. The calculated RRS is shown in Fig.6] Generally, increasing roughness leads to a spectral broadening of the ML peaks, while their number is hardly affected. In the time- 

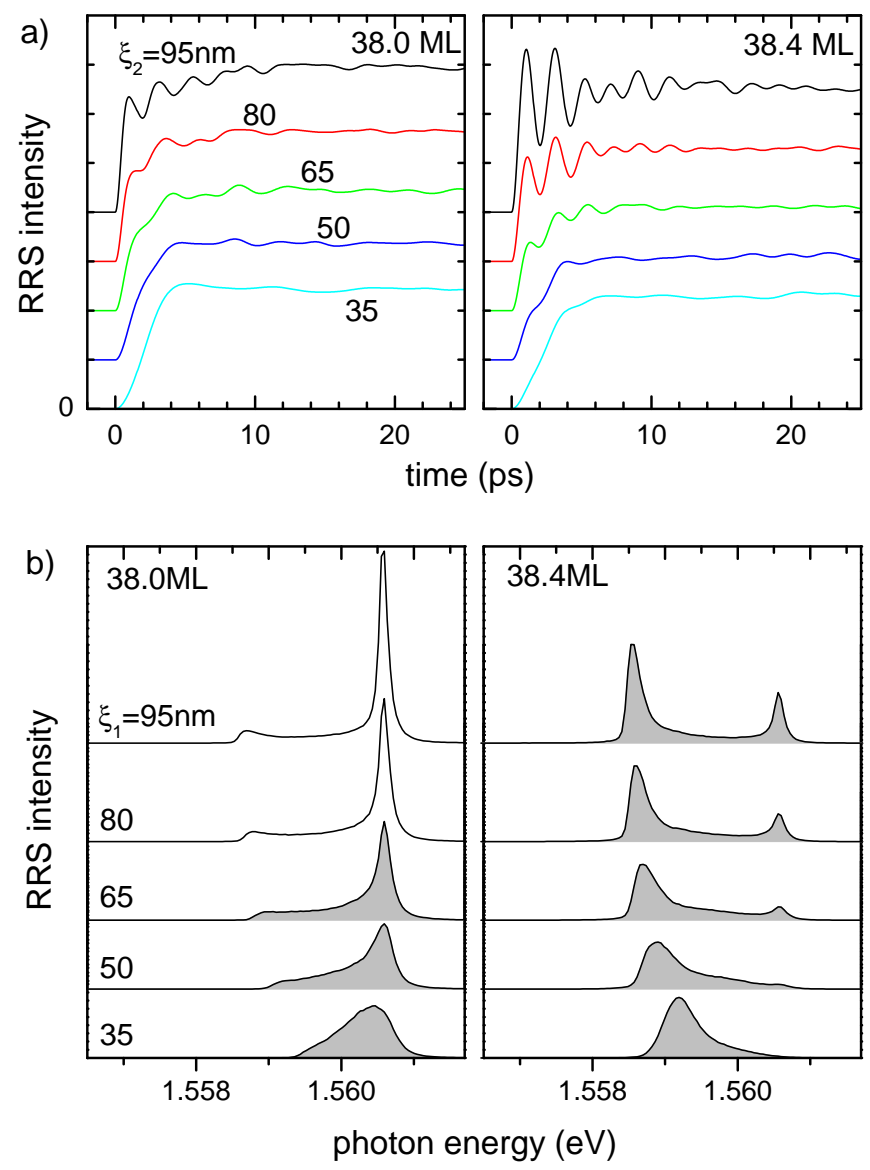

FIG. 7: Calculated time- (a) or spectrally (b) resolved RRS intensity versus the correlation length $\xi_{1}$ for $\xi_{1} / \xi_{2}=0.75$. Results for $\Delta=38.0$ (left) and $\Delta=38.4$ (right) are shown. Data are vertically offset for clarity.

domain, increasing roughness leads to a decrease of the RRS rise time, and to a suppression of the beating between the ML peaks. From the obtained dependency, we

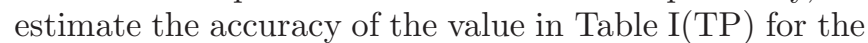
flatness $\zeta_{1}$ to about $\pm 30 \%$.

Let's now look at the influence of the correlation length $\xi_{1}$ while keeping the ratio $\xi_{1} / \xi_{2}=0.75$ constant. The corresponding simulations are displayed in Fig. [7 Increasing this length decreases the lateral quantization energy in the ML islands. At $\xi_{1}=35 \mathrm{~nm}$, the ML peaks are not developed, since the quantization energy is comparable to the ML splitting. With increasing $\xi_{1}$, the ML peaks develop into well defined resonances. In the RRS dynamics, this trend is reflected in the appearance of a well defined temporal beating between the ML peaks. Note the absence of an initial RRS peak predicted for longer correlation length in a simple disorder potential ${ }^{7.9}$. This is related to the perfect interface correlation (compare also with Fig. 国), effectively canceling the long-wavelength components of the disorder. From the obtained dependency, we can estimate the accuracy of the value of the correlation length $\xi_{1}$ in Table प(TP) to about $\pm 15 \%$.
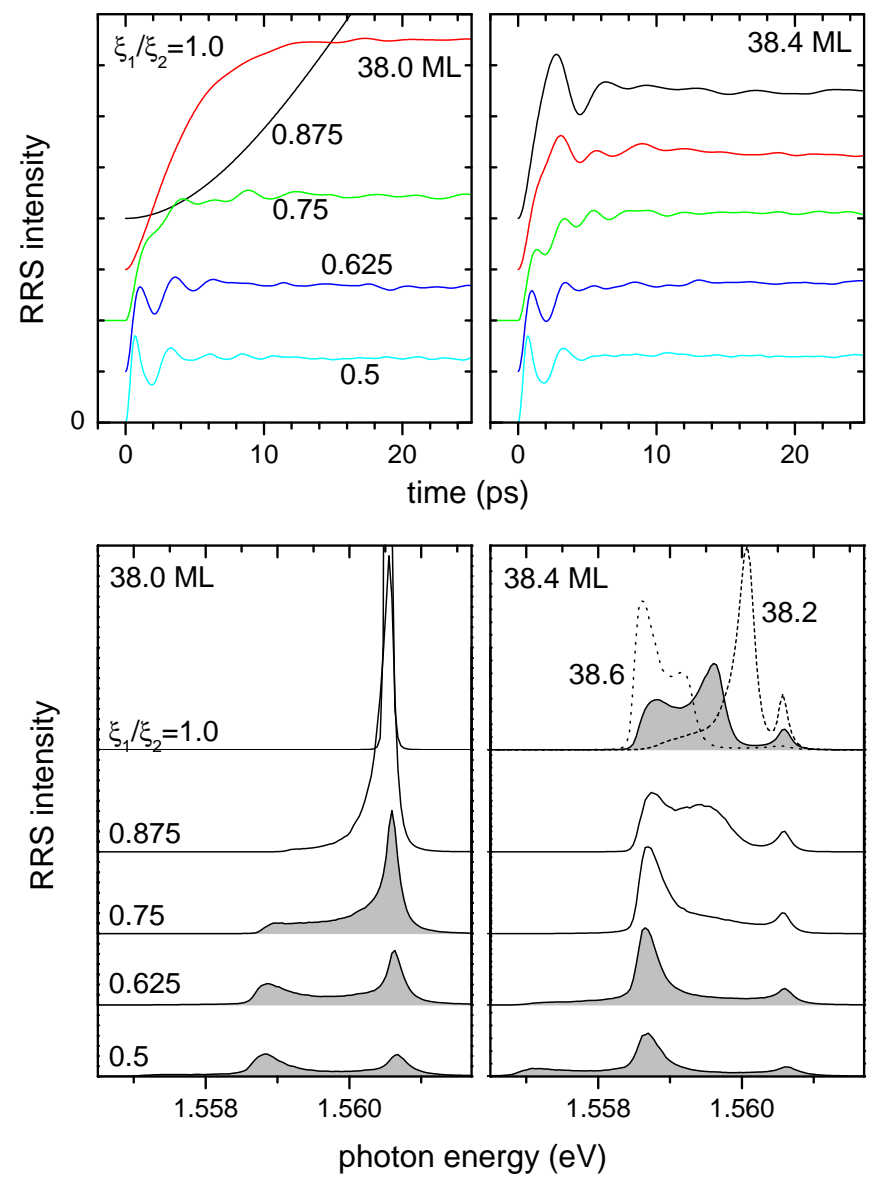

FIG. 8: Calculated time- (a) or spectrally (b) resolved RRS intensity versus the correlation length ratio $\xi_{1} / \xi_{2}$ for $\sqrt{\xi_{1} \xi_{2}}=$ $75 \mathrm{~nm}$. Results for $\Delta=38.0$ (left) and $\Delta=38.4$ (right) are shown. Data are vertically offset for clarity. The dashed and dotted lines in the right lower panel at $\xi_{1} / \xi_{2}=1$ are for $\Delta=38.2$ and $\Delta=38.6$.

Finally, in Fig. 8 we investigate the influence of the ratio of the correlation lengths $\xi_{1} / \xi_{2}$ for a fixed value of $\xi_{1} * \xi_{2}$. For unity ratio, a difference of the interface structure exists in our model only for non-integer ML thickness. For integer $\Delta$ the interface difference is constant, resulting in a spectrally sharp RRS only broadened by the short-range disorder $\sigma$. Varying $\Delta$ continuously from one integer value to the next, rings and stripes of larger thickness form, gradually getting wider until they entirely cover the plane as $\Delta$ reaches its next integer value. The rather well defined ring width leads to a third peak in the spectrum, with an energy above the $39 \mathrm{ML}$ peak given by the one-dimensional quantization energy across the ring. The peak accordingly shifts to lower energy with increasing $\Delta$.

With increasing deviation of $\xi_{1} / \xi_{2}$ from unity, the effect of the interface correlation decreases, so that for $\xi_{1} / \xi_{2}=0.5$ more than two ML peaks are visible, and the RRS dynamics shows an initial peak, similar to the results for decreasing correlation in Fig.[5 and develop- 
ing into the case shown in Fig.4 From the obtained dependency, we can estimate the accuracy of the value in Table凹(TP)for the ratio $\xi_{1} / \xi_{2}$ to about $\pm 8 \%$.

\section{Influence of anisotropy and short-range disorder}

The influence of the correlation length anisotropies within $0.5<\epsilon_{\alpha}<2$ on the simulation results is not very pronounced, and is similar to the combined effects of other parameters. We thus have chosen the isotropic case $\epsilon_{\alpha}=1$ in the following. We remark that an anisotropy is expected due to the surface reconstruction. The effect of such an anisotropy on the exciton fine-structure was observed $^{23}$, and an analysis in terms of a correlated disorder potential in a $5 \mathrm{~nm}$ wide $\mathrm{GaAs} / \mathrm{Al}_{0.3} \mathrm{Ga}_{0.7} \mathrm{As} \mathrm{QW}$ grown without GI revealed 10 a value of $\epsilon \approx 0.6$.

The short range disorder described by $\sigma$ leads to an overall spectral broadening. Its main effect for the parameter set in Table I(TP) is to broaden the sharp ML peaks developing for $\Delta$ close to an integer ML thickness. The present value is $0.8 \mathrm{meV}$. To interpret this value, we consider that the volume over which the exciton averages contains about $N=30000 \mathrm{Ga} / \mathrm{Al}$ sites. For a random distribution of $n \mathrm{Al}$ sites $(n \ll N)$, we would expect ${ }^{54}$ a disorder potential variance of $\sigma=\sqrt{n} E_{c} / N$, where $E_{c}=1.6 \mathrm{eV}$ is the band gap difference between GaAs and AlAs at the $\Gamma$ point. From $\sigma=0.8 \mathrm{meV}$ we estimate an average $\mathrm{Al}$ concentration of $n / N \approx 0.75 \%$. This rough estimate is consistent with the expected segregation 24 .

\section{COMPARISON WITH THE EXPERIMENT}

We now compare the calculated optical properties with the measured RRS and optical density of the sample described as wafer 5 in Ref.13. It contains single GaAs quantum wells, nominally $39,30,23$, and 18 ML wide. The sample was grown by MBE at $630^{\circ} \mathrm{C}$ on a two-inch undoped GaAs [100] wafer using $8 \mathrm{~nm}$ AlAs barriers and $50 \mathrm{~nm}$ GaAs spacers between the wells. This barrier design results in a negligible charging of the QWs because long-lived unpaired charge carriers can tunnel through the narrow AlAs barriers into the GaAs spacers within microseconds. Rotation of the substrate was stopped only during the growth of the wells in order to achieve a continuous variation in well thickness across the wafer (along the [110] direction) while maintaining a constant barrier width. Growth rates were calibrated using reflectance high-energy electron diffraction on a reference wafer. The nominal growth rates were $0.8 \mathrm{ML} / \mathrm{s}$ and 0.3 $\mathrm{ML} / \mathrm{s}$ for GaAs and AlAs, respectively, and a 30\% variation in GaAs growth rate was observed across the wafer. A standard V/III flux ratio of 8 - 10 was used. At each position on the wafer the excitonic resonances of the 4 QWs of different thickness are spectrally well separated, so that by choosing the excitation wavelength we could efficiently select the excitonic response of a single QW.
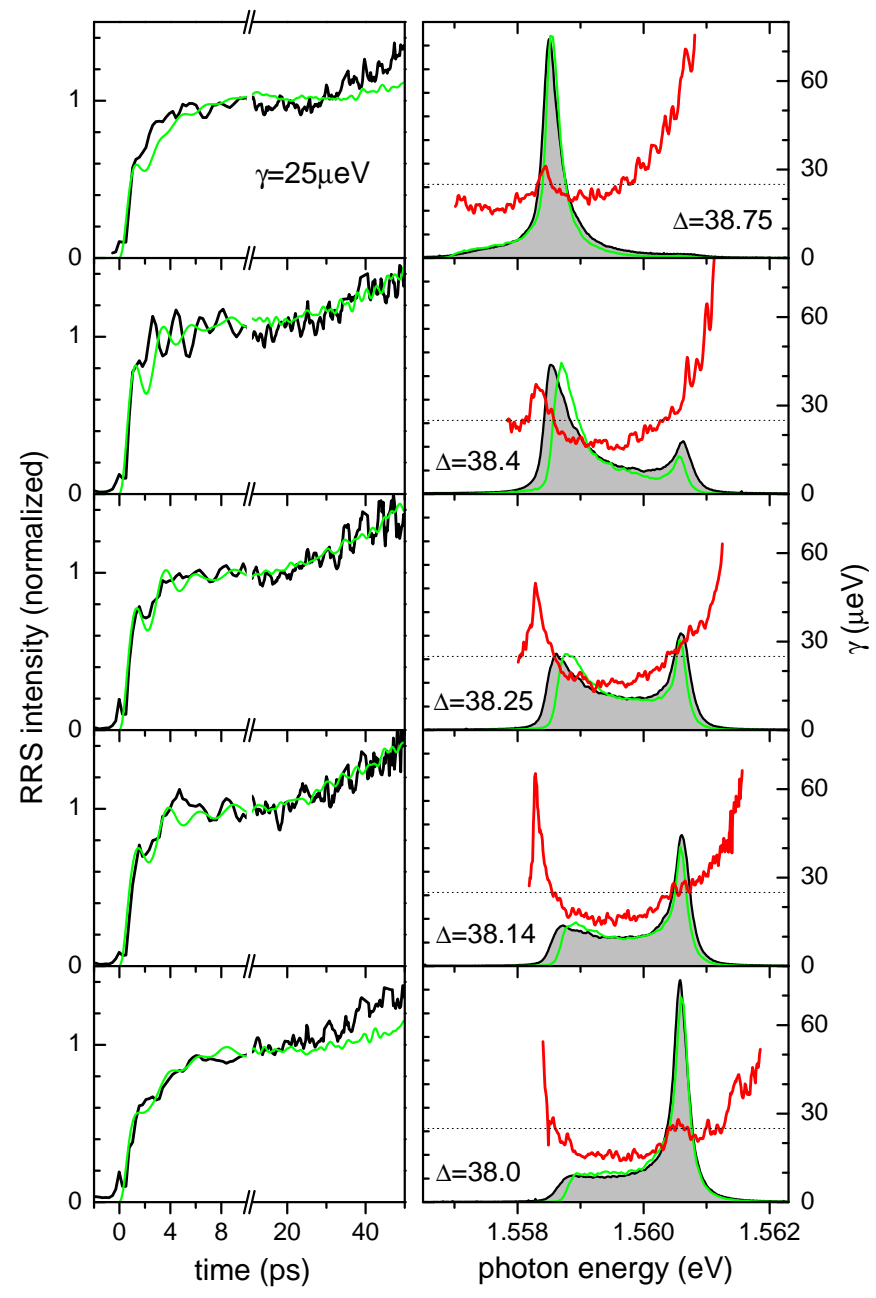

FIG. 9: Spectrally and time-resolved RRS intensity for different QW thicknesses $\Delta$ as indicated. The spectrally resolved RRS is shown on a constant intensity scale for all $\Delta$, together with the homogeneous linewidth (HWHM) $\gamma_{\text {ssa }}(\omega)$ given in red. The time-resolved RRS intensity was measured by spectral interferometry with $300 \mathrm{fs}$ resolution, and is divided through by the exponential decay $\exp (-2 t \gamma)$ using $\gamma=25 \mu \mathrm{eV}$. Note the break in the time axis. The intensity scale is adjusted for each $\Delta$ to the plateau of the data. The statistical error due to the finite number of speckles is $\pm 3 \%$. The simulated RRS using the ES model (see Section IV.C below) and the parameters of Table are superimposed as green lines. The intensities have been scaled to match the measurements, and the time-resolved data have been corrected for an average polarization decay rate of $14 \mu \mathrm{eV}$.

The spectrally resolved RRS intensity was measured using spectral speckle analysis ${ }^{8}$, which also determines the spectrally resolved homogeneous linewidth $\gamma_{\text {ssa }}(\omega)$. The samples were kept at temperatures between $1.5 \mathrm{~K}$ and $50 \mathrm{~K}$. The fundamental hh1-e1 1s exciton resonance was excited by optical pulses from a mode-locked Ti:Sapphire laser of $0.3 \mathrm{ps}$ pulse length. The diameter of the excitation spot was $200 \mu \mathrm{m}$. The excited exciton density was chosen below $10^{9} \mathrm{~cm}^{-2}$ to keep the influence 


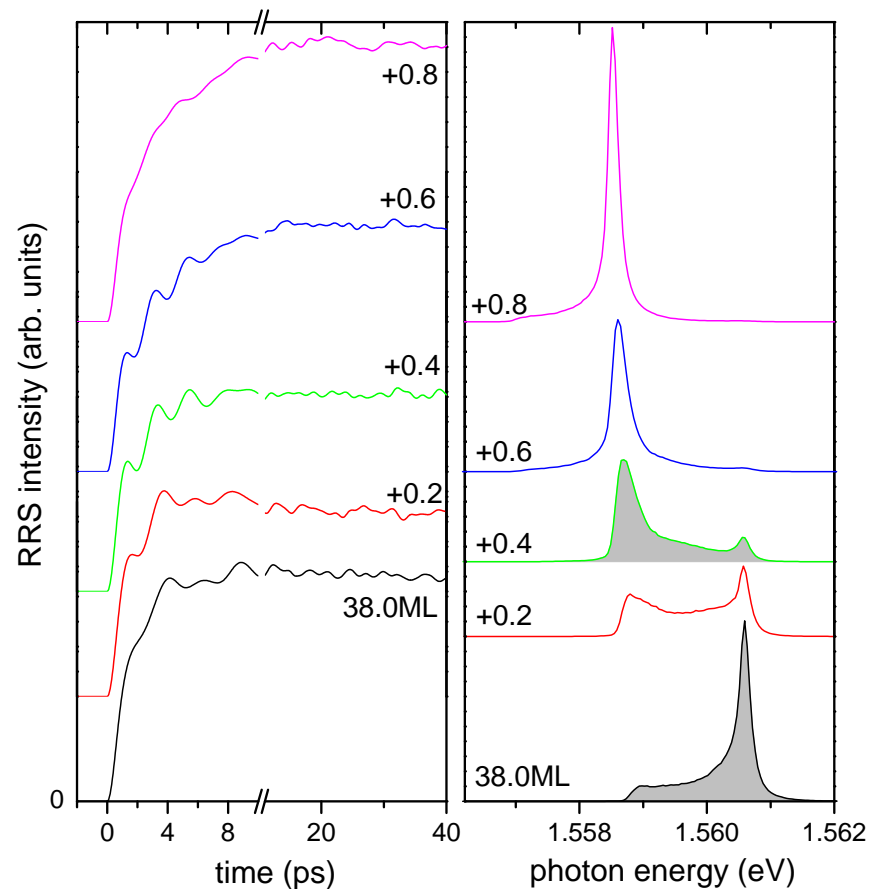

FIG. 10: Calculated time- (left) or spectrally (right) resolved RRS intensity versus $\Delta$ as indicated. Simulation parameters as in table【 Data are offset for clarity.

of the density-dependent broadening by exciton-exciton interaction weak. The secondary emission was passed through an imaging spectrometer and was detected by a nitrogen cooled CCD array with a Voigt spectral response of $20 \mu \mathrm{eV}$ Gaussian and $7 \mu \mathrm{eV}$ Lorentzian full width at half maximum (FWHM). The directional resolution was adjusted to resolve a single speckle, i.e. to be better than the diffraction limit of the excited area on the sample. ${ }^{25}$ All presented data were taken through an analyzer parallel to the linearly polarized excitation impinging on the sample in Brewster angle, in a directional range centered normal to the sample. The time-resolved RRS was measured using passively stabilized spectral interferometry ${ }^{26.27}$ - a simpler approach as compared to actively stabilized versions 28.29 . The time resolution of this measurement is $300 \mathrm{fs}$, while an uncertainty of $\pm 3 \%$ in the measured intensity is introduced by the average over a finite number of speckles. In this case, the diameter of the excitation spot was $100 \mu \mathrm{m}$.

The measurements were taken at a sample temperature of $1.5 \mathrm{~K}$. Different positions on the sample along the thickness gradient were investigated. The measured RRS for varying fractional ML thickness is given in Fig.9 Assuming a linear thickness variation, the observed lateral separation of $3 \mathrm{~mm}$ between equivalent fractional ML positions of 38 and $39 \mathrm{ML}$ thickness was used to calibrate the thickness gradient. The absolute thickness was fixed by comparison of the measured RRS spectra with the simulated ones at integer ML thickness (see Fig.10).

The measured RRS shows the formation of ML peaks as described in the previous sections. The simulated spectra and the dynamics for the parameters of table! are given in Fig.10 and show a general agreement with the measurements. Some discrepancies in the spectral shape and in the energy splitting of the ML peaks are still present for non-integer ML thicknesses. The simulated RRS dynamics reproduces the weak amplitude of the ML oscillations and their suppression close to integer ML thicknesses. The simulated oscillation period is about $10 \%$ longer than in the measurements, related to the discrepancy in the ML peak splitting. The deviations could possibly be reduced by further optimizing the model parameters. However, one qualitative deviation between simulation and experiment is obvious: the presence of a distribution of polarization decay rates $\gamma$ within the exciton ensemble. It manifests itself as a nonexponential decay of the RRS with time, yielding a positive curvature of the RRS dynamics when corrected for a single exponential decay. This non- exponential decay was already noted earlier ${ }^{9}$, and attributed to a distribution of $\gamma$ values within the exciton ensemble. The distribution of $\gamma$ values is even more directly observable in the spectral speckle analysis data, where a spectral dependence of the line-width is found (red curves in Fig. [9].

The distribution of decay rates originates from the inhomogeneous nature of the exciton states. Each localized exciton COM eigenstate is characterized by a different rate of radiative recombination and phonon scattering to other exciton states. To model this distribution, one needs first to compute the exciton eigenstates - a much more computationally demanding task that could be avoided in the first part of this work by solving directly the dynamical RRS equation (1) versus time, with the drawback of having to use a constant value of $\gamma$. Once the exciton eigenstates are known, the distribution of $\gamma$ can be obtained by computing their radiative recombination and acoustic phonon scattering rates in first-order perturbation ${ }^{8.11 .30}$. We have implemented this approach for the presented disorder model, as we discuss in the next section. The resulting simulations are superimposed to the measurements in Fig.9](green curves), and we discuss their comparison with the measurements after introducing the model in the next section.

To evaluate to what extent the present disorder model is applicable to excitons in QWs of different thicknesses in the same sample - which we assume to feature similar interface structures - we have measured the photoluminescence (PL) of the QWs of about 30 and $20 \mathrm{ML}$ thickness

\begin{tabular}{|c|c|c|c|c|}
\hline$\Delta$ & $d F / d \Delta(\mathrm{meV})$ & $E_{\mathrm{B}}(\mathrm{meV})$ & $a_{\mathrm{B}}(\mathrm{nm})$ & $\sigma(\mathrm{meV})$ \\
\hline 38 & 2.1 & 11 & 8 & 0.8 \\
30 & 4.5 & 12.4 & 7.5 & 1.7 \\
20 & 10.5 & 15 & 6.9 & 4 \\
\hline
\end{tabular}

TABLE II: Parameters used in the calculations for different QW thicknesses. 


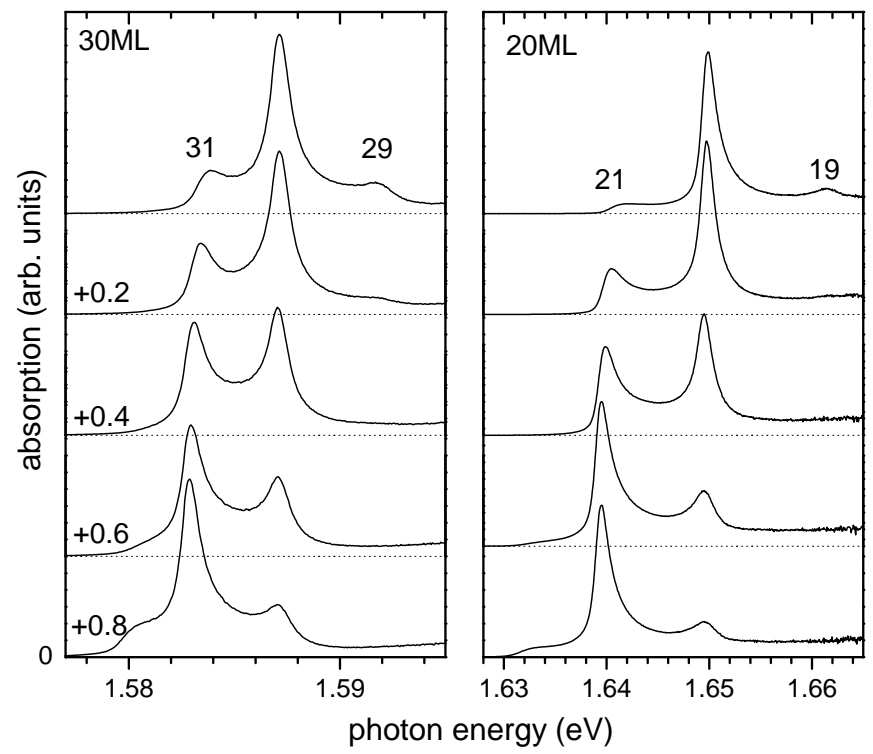

FIG. 11: Measured absorption for $20+$ and $30+$ ML thickness. Data are deduced from photoluminescence at $50 \mathrm{~K}$ lattice temperature assuming exciton thermalization to $55 \mathrm{~K}$.

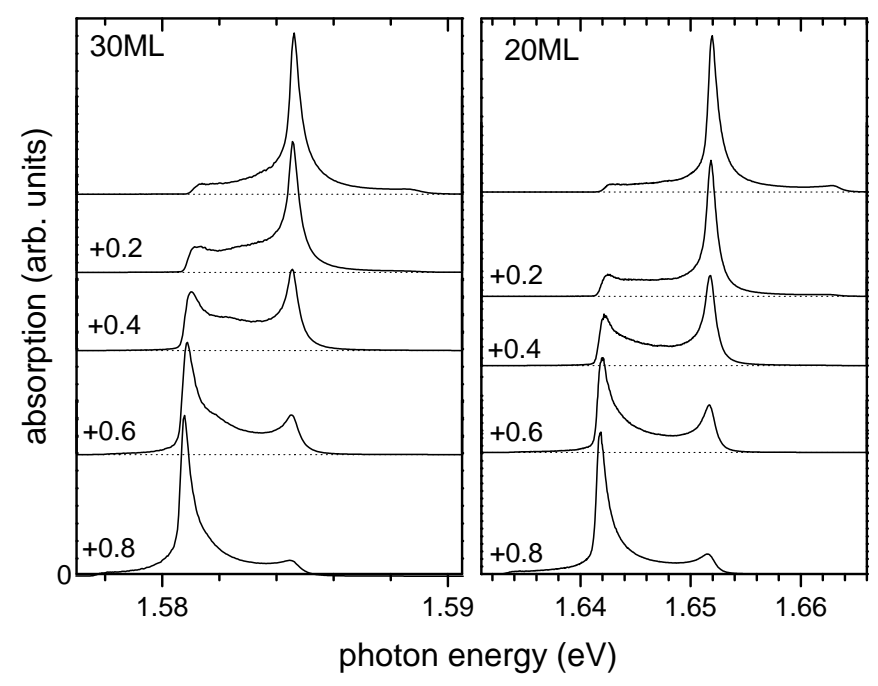

FIG. 12: Calculated absorption for $20+$ and $30+$ ML thickness. Data are offset for clarity.

at a temperature of $50 \mathrm{~K}$. For this elevated temperature, we can assume a thermal equilibrium in the exciton state occupation ${ }^{31}$, which allows us to determine the spectral shape of the absorption in a simple way $\underline{13}$. The resulting data (corrected for the temperature induced band-gap shift of $3 \mathrm{meV}$ ) is shown in Fig.[1] In the absorption, up to 3 different ML peaks are visible. With increasing ML splitting energy, the ML peaks get better defined due to the smaller localization length $\xi_{0}$ compared to the constant interface correlation length. For $20 \mathrm{ML}$ thickness, the exciton continuum absorption is merging with the upper ML peak, a clear sign that the rigid exciton approximation 11 will not be of good accuracy. The ex- citon binding energies $E_{\mathrm{B}}$ for the 30 (20) ML QW are 12 (15) meV, while the ML splittings are 4 (10) meV, so that already for the $30 \mathrm{ML}$ the disorder potential amplitude is close to the exciton binding energy. The exciton binding energies have been determined by a line-shape fit to the absorption of non-GI reference QWs 32 and are in agreement with the values reported in Ref. 33 .

The calculated absorption is given in Fig.12 The exciton Bohr radius $a_{\mathrm{B}}$ was scaled relative to the $38 \mathrm{ML}$ simulation according to $E_{\mathrm{B}}^{-1 / 2}$. All interface parameters are as in table \except $\sigma$, which has been scaled proportional to the ML splitting energy (see Table【). General agreement between simulation and experiment is found. Quantitative differences are narrower lines and less ML peaks in the result of the simulation, compared to experimental data. The homogeneous broadening $\gamma$ at $50 \mathrm{~K}$ can be estimated to be below $200 \mu \mathrm{eV}$, and should thus not be the main origin of the different line-widths in theory and experiment.

\section{MICROSCOPIC TREATMENT OF PHONON SCATTERING AND RADIATIVE DECAY}

We now describe the theoretical approach used to simulate the RRS signal accounting for a distribution of the polarization decay rates. We consider two physical mechanisms. First, the radiative decay of each localized exciton. Second, the polarization decay due to scattering between different exciton states assisted by acoustic phonon emission or absorption. The details of how both rates are modeled for exciton COM eigenstates are described in Ref.11. The effect of a radiative renormalization of the states that results e.g. in a polarization splitting ${ }^{10.23}$ is neglected. The typical splitting energies are similar to the radiative decay rates. The splitting can lead to an additional slow dynamics of the RRS due to polarization beating, mainly relevant on a timescale longer than that considered in the present analysis.

In order to compute the rates it is necessary to know the eigenstates and energy eigenvalues of the exciton COM motion within the disorder potential. These are obtained by solving the eigenvalue problem given by the COM Schrödinger equation (1), which reads

$$
\left(-\frac{\hbar^{2}}{2 M} \nabla^{2}+V(\mathbf{R})\right) \psi_{j}(\mathbf{R})=E_{j} \psi_{j}(\mathbf{R})
$$

where $\psi_{j}(\mathbf{R})$ are the COM eigenfunctions and $E_{j}$ the corresponding energy eigenvalues. Eq. (9) is solved numerically on the same grid used for simulating the timedependent equation (11). When neglecting decay rates, we can express exactly the Green's function of Eq. (11) in terms of its eigenvalue decomposition

$$
G\left(\mathbf{R}, \mathbf{R}^{\prime}, t\right)=\sum_{j} \psi_{j}^{*}\left(\mathbf{R}^{\prime}\right) \psi_{j}(\mathbf{R}) \exp \left(-\frac{i E_{j}}{\hbar} t\right)
$$


From this Green's function, the solution $P(\mathbf{R}, t)$ of (1) for an arbitrary input field $E_{\text {in }}(\mathbf{R}, t)$ can be computed. The advantage of this approach is that we can generalize the solution by introducing decay rates $\gamma_{j}$ for each exciton COM eigenstate. The corresponding COM Green's function can be rewritten as

$$
G\left(\mathbf{R}, \mathbf{R}^{\prime}, t\right)=\sum_{j} \psi_{j}^{*}\left(\mathbf{R}^{\prime}\right) \psi_{j}(\mathbf{R}) \exp \left(-\frac{i E_{j}}{\hbar} t-\gamma_{j} t\right) .
$$

This expression can no longer be related to the solution of Eq. (11). However, as we will see later in this section, once the decay rates have been accurately modeled, it gives a better account of the observed RRS dynamics. As for the time-propagation simulations we use a delta-pulse excitation field at $\mathbf{k}_{\text {in }}=0, E_{\text {in }}(\mathbf{R}, t)=E_{\text {in }} \delta(t)$, corresponding to an excitation beam impinging on the sample at normal incidence. For this configuration, the RRS amplitude resulting from the simulation can be rewritten as

$$
E_{\text {out }}(\mathbf{k}, t)=\sum_{j} \psi_{j}^{*}(\mathbf{k}) \psi_{j}\left(\mathbf{k}_{\text {in }}\right) \exp \left(-\frac{i E_{j}}{\hbar} t-\gamma_{j} t\right),
$$

where

$$
\psi_{j}(\mathbf{k})=\frac{1}{\sqrt{A}} \int d \mathbf{R} \psi_{j}(\mathbf{R}) \exp (i \mathbf{k} \cdot \mathbf{R}),
$$

is the Fourier transform of the exciton COM eigenfunction, $A$ being the simulation area.

\section{A. Radiative rates}

The radiative polarization decay rates $r_{j}$ are computed from the dipole matrix element, according to the Fermi golden rule. Following Zimmermann et al ${ }^{11}$ they are

$$
r_{j}=\frac{\Gamma_{0}}{A} \sum_{\mathbf{k}} \frac{\Theta\left(k_{0}^{2}-k^{2}\right)}{k_{0} \sqrt{k_{0}^{2}-k^{2}}}\left(k_{0}^{2}-k^{2} / 2\right)\left|\psi_{j}(\mathbf{k})\right|^{2} .
$$

Here $A$ is the area of the simulation domain, $\mathbf{k}$ is as usual the in-plane wavevector, whereas $k_{0}=\sqrt{\epsilon_{b}} E_{x} /(\hbar c)$ is the wavevector corresponding to the light cone, defining the boundary of the in-plane momentum region where an exciton state can decay into a photon mode radiating in the $z$-direction. Because of energy and momentum conservation, excitons at $k>k_{0}$ result into a surface polariton mode characterized by an evanescent light wave in the vertical direction. The quantity $\Gamma_{0}$ is the radiative rate of a plane-wave exciton state having zero COM momentum. Expression (14) has been averaged over the azimuthal angle and over the two possible polarization directions of the emitted electromagnetic field. This average is a good approximation, despite the irregularly shaped COM eigenfunctions, as the eigenstates relevant to the optical response are localized on a spatial range much shorter than the optical wavelength in the medium, which for
GaAs is in the range of $250 \mathrm{~nm}$. Consequently, the wavefunctions are approximately isotropic for $k<k_{0}$. For the numerical calculations, we have used $\Gamma_{0}=34 \mu \mathrm{eV}$, which is estimated ${ }^{34}$ for a $11 \mathrm{~nm}$ GaAs/AlAs QW heavy-hole exciton having an exciton binding energy of $11 \mathrm{meV}$.

\section{B. Phonon rates}

The phonon scattering rates are computed accounting for the exciton deformation potential interaction with longitudinal acoustic phonons. A detailed account of this calculation is again found in the Review by Zimmermann et $a l^{11}$ and we report here just the essential expressions. Fermi golden rule gives the following expression for the polarization scattering from state $j$ to state $l$ by emission or absorption of a phonon

$$
\begin{array}{r}
p_{l j}=\frac{2 \pi}{\hbar} \sum_{\mathbf{q}}\left|t_{l j}^{\mathbf{q}}\right|^{2}\left[\left(n_{q}+1\right) \delta\left(E_{l}-E_{j}+\hbar \omega_{q}\right)\right. \\
\left.+n_{q} \delta\left(E_{l}-E_{j}-\hbar \omega_{q}\right)\right],
\end{array}
$$

where $\mathbf{q}$ is the phonon momentum in three dimensional reciprocal space, $n_{q}$ is the Bose thermal distribution of phonons, $\omega_{q}=v_{s} q$ is the phonon dispersion, $v_{s}$ being the sound velocity. The quantity $t_{l j}^{\mathrm{q}}$ is the scattering matrix element of the deformation potential Hamiltonian which, after introducing the factorization ansatz between relative and $\mathrm{COM}$ exciton motion, is given by

$$
\begin{array}{r}
t_{l j}^{\mathbf{q}}=\sqrt{\frac{\hbar \omega_{q}}{2 v_{s}^{2} \rho_{m} V}}\left(\psi_{j} \psi_{l}\right)_{\mathbf{q}_{||}}\left[D_{e} K_{e}\left(q_{z}\right) \chi\left(q_{||} / \eta_{e}\right)\right. \\
\left.+D_{h} K_{h}\left(q_{z}\right) \chi\left(q_{||} / \eta_{h}\right)\right] .
\end{array}
$$

Here, $\rho_{m}$ is the mass density of the material, $D_{\alpha}$ are the deformation potentials for electron and hole, $q_{z}$ and $\mathbf{q}_{\|}$ are respectively the $z$ and in-plane components of the phonon momentum, and $\eta_{\alpha}=\left(m_{e}+m_{h}\right) / m_{\alpha}$ are mass factors. The functions $K_{\alpha}$ and $\chi$ are overlap integrals between the phonon wavefunction and, respectively, the confinement functions in the $z$ direction and the relative $1 s$ exciton wavefunction. The expression

$$
\left(\psi_{j} \psi_{l}\right)_{\mathbf{q}_{\|}}=\int d \mathbf{R} \psi_{j}^{*}(\mathbf{R}) \exp \left(-i \mathbf{q}_{||} \cdot \mathbf{R}\right) \psi_{l}(\mathbf{R}),
$$

is the corresponding overlap integral for the in-plane COM wavefunctions. After summing Eq. (16) over the $q_{z}$ variable we find for the scattering rates

$$
p_{l j}=\frac{2 L_{z}}{\hbar^{3} v_{s}^{2}}\left|\Delta E_{j l}\right| n\left(\Delta E_{j l}\right) \sum_{\mathbf{q}_{||}} \frac{\left|t_{l j}^{\mathbf{q}_{\mid l}, \bar{q}_{z}}\right|^{2}}{\bar{q}_{z}},
$$

where $\Delta E_{j l}=E_{l}-E_{j}, \bar{q}_{z}=\sqrt{\left(\Delta E_{j l} / \hbar v_{s}\right)^{2}-q_{\|}^{2}}$ is the restriction imposed by energy-momentum conservation, $L_{z}$ is the size of the integration domain in the $z$ 

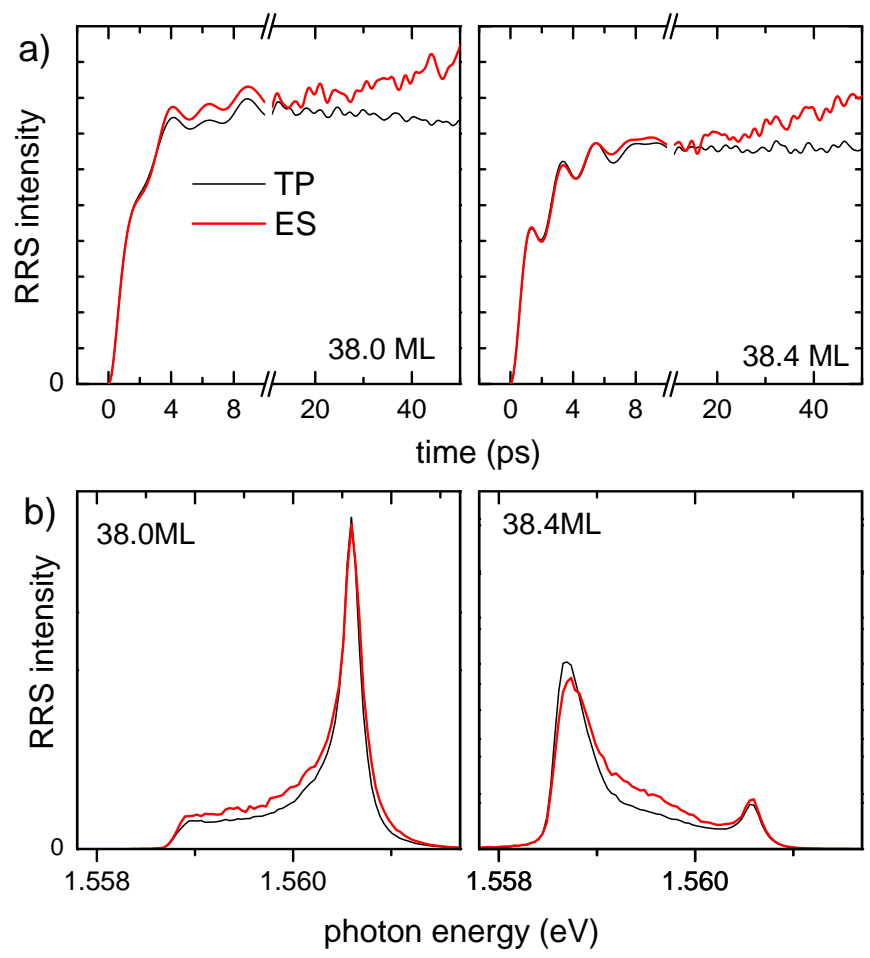

FIG. 13: Calculated time- (a) or spectrally (b) resolved RRS intensity using the time propagation (black) and the eigenstate calculation (red). The eigenstate dynamics has been corrected for an average polarization decay rate $\gamma=14 \mu \mathrm{eV}$. Note the break in the time axis. The spectrally resolved data are normalized to the spectrally integrated intensity of the 38.0 ML data. Disorder parameters are given in table

direction (which is canceled when plugging in the explicit expression for the scattering matrix element), and $n(E)=\left(\exp \left(E /\left(k_{B} T\right)-1\right)^{-1}\right.$ is the Bose energy distribution.

The overall decay rate for a given exciton state $j$ is then given by the sum of its radiative decay rate and all out-scattering phonon rates $p_{l j}$

$$
\gamma_{j}=r_{j}+p_{j}, \quad p_{j}=\sum_{l} p_{l j},
$$

where we have denoted the overall phonon outscattering rate of the $j$-th state by $p_{j}$. For the simulations, we use the deformation potentials values $D_{e}=7 \mathrm{eV}, D_{h}=3.5$ $\mathrm{eV}$, the mass density value $\rho_{m}=5.37 \mathrm{~g} \mathrm{~cm}^{-3}$, and the sound velocity $v_{s}=5.33 \times 10^{5} \mathrm{~cm} \mathrm{~s}^{-1}$, typical for GaAs bulk material ${ }^{35}$ The electron and hole effective masses were respectively $m_{e}=0.07$ and $m_{h}=0.18$ times the bare electron mass.

\section{Simulations: Ensemble averages}

We can now compare the results of the ES calculation (Eq. (12)) with the one of the TP calculation (Eq.11). Using the time-propagation disorder potential parameters
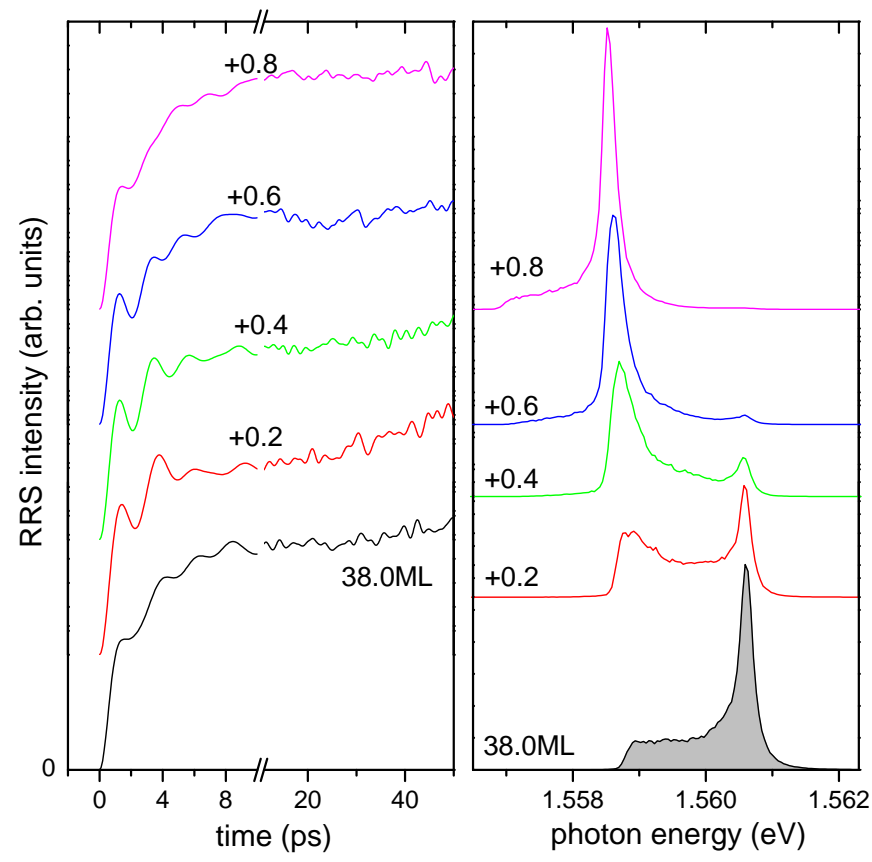

FIG. 14: Calculated time- (left) or spectrally (right) resolved RRS intensity versus $\Delta$ as indicated. The RRS dynamics has been corrected for an average polarization decay rate $\gamma=$ $14 \mu \mathrm{eV}$. Eigenstate simulation with parameters as in Table! Data are offset for clarity.

given in Table we show in Fig. 13 the time-resolved and spectrally resolved RRS intensity for $\Delta=38.0$ and 38.4 ML calculated with both methods. The timeresolved RRS calculated in the TP and ES model are nearly identical at early times $t<10 \mathrm{ps}$. This is because all relevant decay rates $\gamma_{j}$ are much smaller than the inhomogeneous broadening, so that the nonuniform polarization decay among the eigenstates is irrelevant $\left(\exp \left(-t\left(\gamma_{j}-\gamma\right)\right) \approx 1\right)$, and the RRS dynamics is determined by the energy-level distribution associated with the disordered exciton eigen-states ${ }^{9}$. For later times, the energy-level distribution creates a constant RRS signal, given by the incoherent sum of the intensities emitted by the individual exciton states, as can be seen in the $\mathrm{TP}$ results. In this regime the decay rate distribution is relevant since $\exp \left(-t\left(\gamma_{j}-\gamma\right)\right)$ deviates significantly from unity, yielding a non-exponential RRS decay. The deviation from a single exponential decay in the ES calculation is similar to the one measured in the time-resolved RRS (Fig.9), indicating that the ES model is describing its main physical origin.

Comparing the spectra of the TP and the ES calculation, some difference are apparent, especially for energies between the ML peaks - the ES calculation tends to result in more spectral broadening. This difference relates to the distribution of phonon and photon related dephasing rates. Exciton states energetically in between the ML peaks are likely to be excited states of a ML trench, which have rather small radiative rates. Since 
their phonon scattering rates are still smaller than the average radiative rates, their dephasing rate is smaller than average, resulting in a longer and thus spectrally stronger RRS emission as compared to the TP result. To reproduce the experimentally measured RRS spectra with the ES calculation, we needed therefore to adjust the disorder model parameters slightly, as detailed in the ES row of table凹 The resulting calculated RRS spectra and dynamics are given in Fig.14 and show a general agreement with the measurements. However, the calculated average polarization decay rate of $14 \mu \mathrm{eV}$ is lower than the measured one of $25 \mu \mathrm{eV}$. Such a deviation was noticed already in previous works 8 , and can have different origins. On the theoretical side, the deformation potentials, which enter the phonon scattering rates quadratically, are only known up to a factor of two. The radiative rate $\Gamma_{0}$ can be influenced by the dielectric surrounding including dipole-dipole interaction between the exciton states, and also directly by the disorder potential if the rigid exciton approximation breaks down ${ }^{11,36}$ resulting in a modified electron-hole overlap. On the experimental side, there could be an influence of residual exciton-exciton scattering, the influence of spectral diffusion ${ }^{37}$, and also a systematic error due to the finite spectral resolution. Measurements of the dephasing time of exciton ensembles in similar structures by four-wave mixing ${ }^{38}$ showed a polarization decay comparable to the one measured here. We have made no attempt to modify the model parameters towards a better agreement with the experiment since we are mainly concerned with the effect of the disorder potential in this work.

Having at hand the exciton eigenstates with their radiative emission and phonon-assisted relaxation rates, we can investigate in detail their properties at different energies in the disorder potential. We do this in the following for $\Delta=38.0$ and 38.4 ML. The calculated spectral distributions of the density of states (DOS) and the phonon and radiative rates for low temperature $(\mathrm{T}=1.5 \mathrm{~K})$ are given in Fig.15] The exciton DOS $D_{\mathrm{X}}(\omega)$ is compared in the top panel to the normalized spatial potential density $D_{V}(\omega)$. For long potential correlation lengths, the lateral quantization energies are negligible and $D_{\mathrm{X}}(\omega)$ should be just given by the convolution of $D_{V}(\omega)$ with the $2 \mathrm{D}$ exciton DOS $\theta\left(\hbar \omega-E_{0}\right) \frac{M}{\pi \hbar^{2}}$ :

$$
D_{\mathrm{X}}(\omega) \approx \frac{M}{\pi \hbar} \int_{\omega^{\prime}=-\infty}^{\omega} D_{V}\left(\omega^{\prime}\right) d \omega^{\prime}
$$

This general behavior is visible also in the calculated data, but due to the finite lateral quantization energies, an additional blueshift of $D_{\mathrm{X}}(\omega)$ is observed.

The distributions of the scattering rates are more interesting, as they provide information about the character of the states. At a temperature of $1.5 \mathrm{~K}$, the thermal energy $k_{\mathrm{B}} T \approx 0.1 \mathrm{meV}$ is much smaller that the ML splitting energy of about $2 \mathrm{meV}$, so that phonon absorption processes are not significant. In this case, the phononassisted transitions are directed towards lower-lying exciton states with significant spatial overlap. In a ML
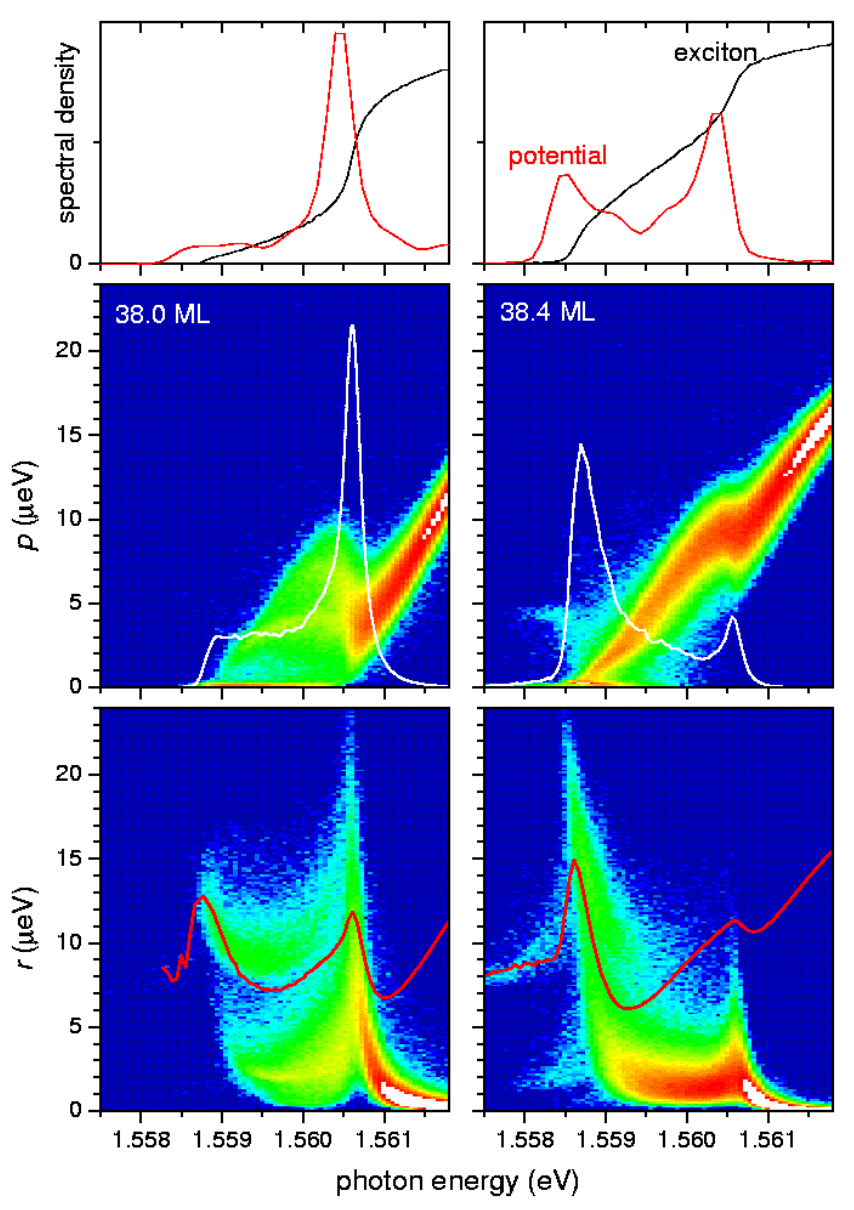

FIG. 15: Probability distribution of phonon scattering rates $D_{p}(p, \omega)$ and radiative decay rates $D_{r}(r, \omega)$ for exciton states in a QW of 38.0 and 38.4 ML thickness. Other parameters as in Table【 Logarithmic color scale over three decades. The RRS spectra are superimposed as white lines, and the average decay rate $\gamma(\omega)$ as red lines. The top plots show the exciton density of states $D_{\mathrm{X}}(\omega)$ and the potential density $D_{V}(\omega)$.

island, the local exciton ground state has thus a negligible $p$, while the first excited state acquires some $p$, and the second excited state even more. The radiative coupling shows the opposite behaviour: The ground state has a nodeless wavefunction and thus a large $k<k_{0}$ component, resulting in a large $r$ (see Eq. (14)). Excited states have additional nodes in the wavefunction, and thus suppressed $k<k_{0}$ components. The calculated distributions $D_{p}(p, \omega)$ and $D_{r}(r, \omega)$ of $p$ and $r$, respectively, are shown in the lower four panels of Fig.[15. For $\Delta=38.0$, the $39 \mathrm{ML}$ regions have only a small spatial coverage, and thus form sub-wavelength sized trenches surrounded by $38 \mathrm{ML}$ regions. Most exciton states in the spectral region below the $38 \mathrm{ML}$ potential energy $F(38)$ are thus localized in individual trenches of $39 \mathrm{ML}$ thickness and show the discussed behaviour. The states of lowest energy $(\omega<1.559 \mathrm{eV})$ are dominantly ground states, showing a large $r$ and vanishing $p$. In the re- 
gion $1.559 \mathrm{eV}<\omega<1.560 \mathrm{eV}$, the first two excited states (p-like) show up, with a 3-5 times reduced $r$, but a significant $p \approx 3 \mu \mathrm{eV}$. For $1.5596 \mathrm{eV}<\omega<1.5605 \mathrm{eV}$, also higher excited states are present as indicated by a feature of $D_{p}(p, \omega)$ at $p \approx 7 \mu \mathrm{eV}$, and a broadening of $D_{r}(r, \omega)$ of the excited states. The ground states show a non-monotonic dependence of $r$ on the transition energy: decreasing with increasing energy up to halfway to the energy of the $38 \mathrm{ML}$ barrier, and then increasing again. This can be understood considering the corresponding evolution of the wavefunction extension: The state energy separation to the $39 \mathrm{ML}$ energy $(1.5585 \mathrm{eV})$ can be interpreted as quantization energy $E_{Q}$ of the state in the trench. For infinite barriers and a square trench of size $L$, we find $E_{\mathrm{Q}}=(\pi \hbar / L)^{2} / M$, yielding $0.3 \mathrm{meV}$ for $L=100 \mathrm{~nm}$. Small quantization energies thus correspond to large trench sizes with large wavefunctions having large $k<k_{0}$ components. With decreasing trench size, the wavefunction decreases in size, until $E_{\mathrm{Q}}$ reaches about half the barrier height. With further decrease in trench size, the increasing penetration of the wavefunction into the lateral barrier leads to an increase of the wavefunction size and thus of the $k<k_{0}$ component. This behaviour was previously predicted ${ }^{39.40}$ and experimentally verified 41 .

The energy-dependent averaged linewidth

$$
\gamma(\omega)=\left\langle\gamma_{j}\right\rangle_{E_{j}=\hbar \omega}
$$

is given in the lower panel of Fig.15] It shows a dependence similar to the one measured by spectral speckle analysis (see Fig. 9), with maxima close to the integer ML energies due to the large radiative rates of the corresponding weakly confined exciton states. In this comparison, we have to keep in mind that the weighting of the states in the average linewidth determined by spectral speckle analysis ${ }^{8.42 .43}$ is not just uniform, as assumed in Eq. (21), but each state is weighted by its RRS intensity, scaling like $r^{2} /(r+p)$. This weighting leads to a measured dephasing which is typically by some $10 \%$ larger that the unweighted average $\gamma(\omega)$. Yet, this weighting cannot explain the observed factor of two between experiment and prediction.

For a direct quantitative comparison of the simulated RRS spectra and dynamics with the measurements, we show in Fig.9 the ES simulation results for the exact ML thicknesses of the measurements superimposed on the measured data. The comparison shows good overall quantitative agreement, in the general shape of both spectral and time-resolved data and, particularly, in the amplitude of the beatings in the time-resolved data at non-integer ML thickness. We find the following deviations significantly above the statistical noise, which we believe are not related to a lack of fine-tuning of the model parameters:

i) The oscillation beat frequency and the splitting of the monolayer energy are about $10 \%$ smaller in the simulation, even though the energy shift due to a thickness change of one ML is perfectly reproduced. In the model, the smaller splitting for non-integer ML thickness is a natural consequence of the lateral quantization in the islands of finite size. It is also instructive to observe that this effect is less pronounced in the simulation of the thinner QWs (see Fig.12) since the in-plane size of the ML islands is constant, while the ML splitting increases as the QW thickness decreases. In the corresponding experiment instead (Fig. [11), the effect is more pronounced. One possible origin of this deviation could be the rigid exciton approximation. Removing this approximation, exciton states localized in the small monolayer islands would display a larger electron-hole coulomb interaction ${ }^{36}$, leading to a shift of the lower ML peak to lower energy. Unfortunately it is numerically prohibitive to perform the necessary ensemble averages without this approximation. The observed deviation of about $0.3 \mathrm{meV}$ is a small correction relative to the exciton binding energy of $11 \mathrm{meV}$, and lies within the expected magnitude of this effect. Another possible source of discrepancy are finer details of the heterointerface structure that are not accounted for in our disorder model. For example a nonGaussian spatial correlation could give rise to a larger spread of island sizes, which reduces the lateral quantization in the largest islands. Extending the model in such direction is a possible matter of future development, but we feel it should be supported by more extensively sampled experimental data.

ii) The long-time nonexponential RRS dynamics is not well reproduced close to integer ML thickness. Generally, this dynamics is given by the distribution of dephasing rates across the state ensemble, and also, since we excite and detect light linearly polarized along [110], by the distribution of the fine-structure splitting and its orientation ${ }^{10}$. We have discussed the dephasing rate distribution already in the previous paragraph. The finestructure splitting would lead to an initial decay of the signal within a time given by the inverse average splitting energy, typically in the $20-50 \mathrm{ps}$ regime $\frac{10}{\underline{0}}$. This would result in a stronger non- exponential RRS decay, similar to the deviation observed. The reduced importance of this effect for non-integer ML thickness could be due to the alignment of the fine-structure along the [110] direction in the (anisotropic) ML islands. A numerical calculation of the fine-structure splitting in the present model is only feasible in a diagonal approximation in the eigenstates, due to computational limitations. This extension of the model, together with a polarization direction dependent RRS dynamics measurement, could be an interesting future investigation.

\section{Simulations: Single realizations}

Having described in the previous section ensemble averaged properties, we turn now to individual excitonic states in specific disorder realizations. These results are important for comparison with the spectroscopy of individual excitonic states performed using high spatial 

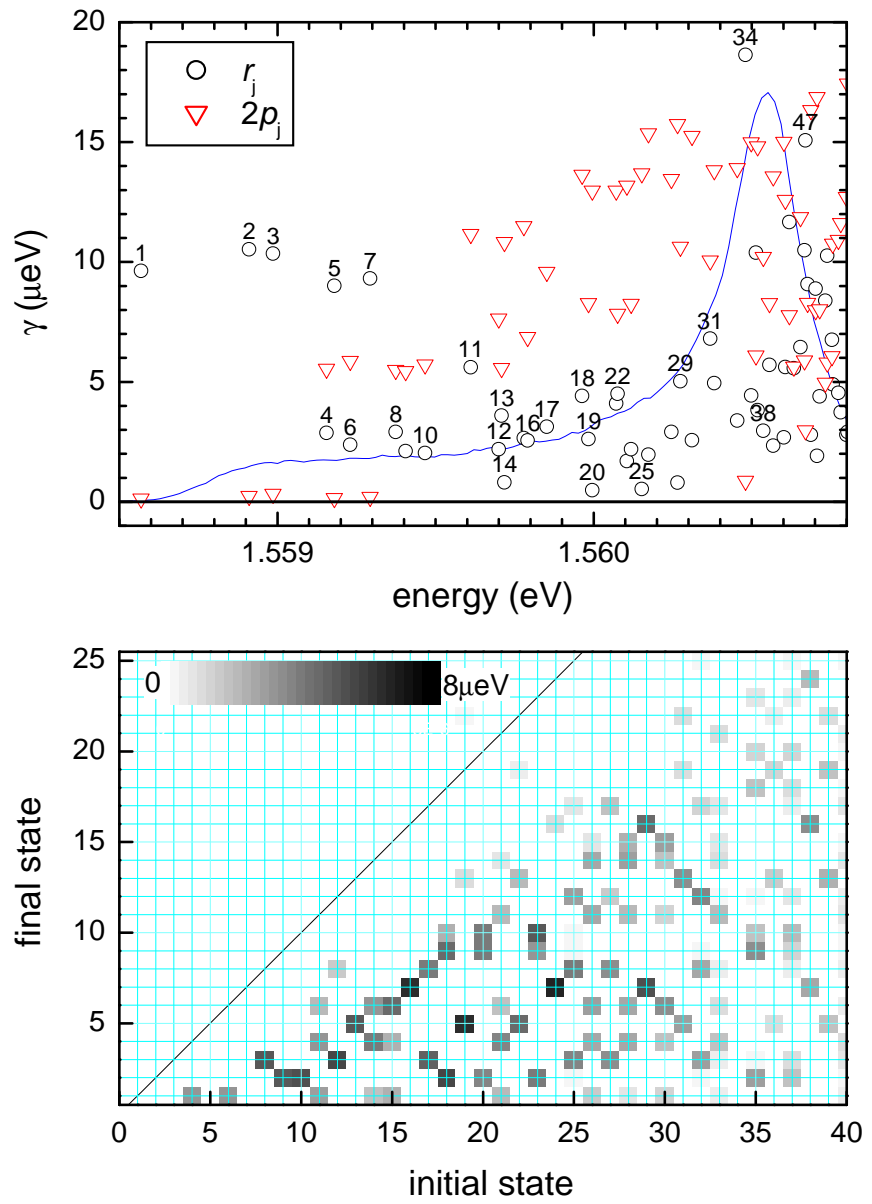

FIG. 16: Excitonic states in a single disorder potential realization $V_{\mathrm{I}}(\mathbf{R})$ of $(500 \mathrm{~nm})^{2}$ with periodic boundary conditions, using the parameters of table $\llbracket$ and $\Delta=38.0$. Top: Phonon scattering $\left(p_{j}\right)$ and radiative decay rates $\left(r_{j}\right)$ of the exciton states $j$ with energy $E_{j}$. The numbered states $j=1,2, \ldots$ have ascending energy $\left(E_{j}<E_{j+1}\right)$. The ensemble averaged RRS spectrum is given (blue line) for comparison. Bottom: Phonon scattering rates $p_{l j}$ from the state $j$ to the state $l$ at $\mathrm{T}=1.5 \mathrm{~K}$. Linear grey scale as displayed.

resolution $44,45,46$. The simulation area used in our calculations of $(500 \mathrm{~nm})^{2}$ is comparable to the typical sizes of the regions probed in this kind of experiments, so that the measured and simulated exciton state ensembles are similar.

We have chosen the thickness $\Delta=38.0$ for the simulations as for this fractional thickness well defined trenches of $39 \mathrm{ML}$ thickness are formed in the $38 \mathrm{ML}$ plateau. Two realizations I \& II of the disorder are discussed in the following, having trenches of more circular or more elongated and irregular shape, respectively. The simulated potential landscapes $V_{\mathrm{I}, \mathrm{II}}(\mathbf{R})$ are shown in the top left panel of Fig.17 and Fig.19] The remaining panels of Fig.17 and Fig.19 display the square modulus of some exciton COM wavefunctions selected among the lowest energy eigenstates. Each of these states is, in general, well localized in a local minimum of the disorder po-

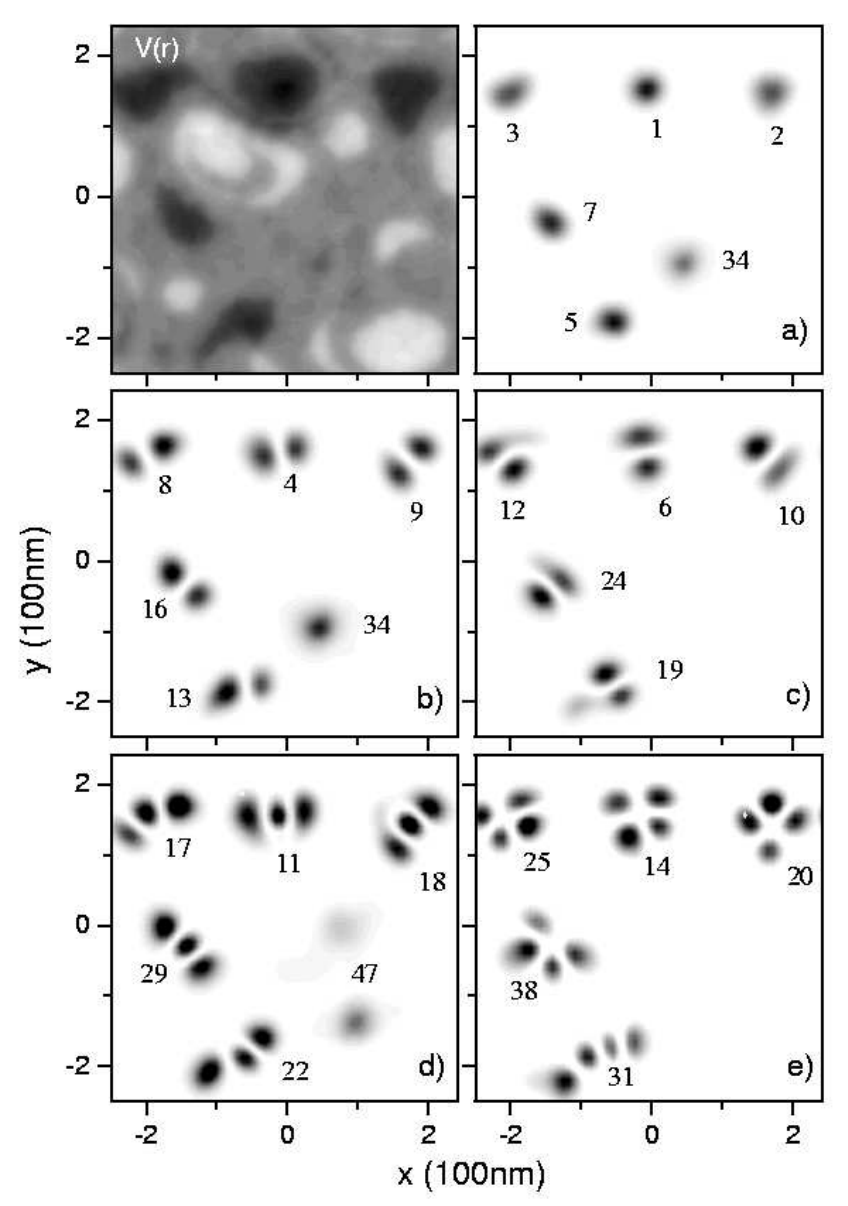

FIG. 17: Disorder potential $V_{\mathrm{I}}(\mathbf{R})$ (top left) on a scale from 1.5575 (white) to $1.5635 \mathrm{eV}$ (black). a)-e) Probability distributions $\left|\Psi_{\alpha}\right|^{2}$ of selected excitonic states as labeled. The grey scale is from zero (white) to a) 800, b,c) 500 and d,e) $300 \mu \mathrm{m}^{-2}$ (black).

tential. We have therefore chosen to plot in each panel several states having similar features, according to the discussion below. In the plots, these excitonic states are labeled by an eigen-state number $j$, sorted in order of ascending eigen-energy $E_{j}$. Each state is characterized by its radiative and phonon scattering rate $r_{j}$ and $p_{j}$, which are plotted in Fig.[16] and Fig.18] respectively. From the mutual phonon scattering rates $p_{l j}$ we can identify corresponding ground and excited states of individual trenches.

We start the discussion with realization I. The state 1 is the global exciton ground state of the system and thus also a local ground state. It shows a large $r \approx 10 \mu \mathrm{eV}$ (corresponding to a dipole moment $\mu \approx 105$ Debye as given by $\mu=\sqrt{6 \pi \hbar r \epsilon_{0} c^{3} n^{-1} \omega^{-3}}$ using the refractive in$\operatorname{dex} n \approx 3.5$ ), and negligible phonon scattering $p_{1}$, as expected. States 2,3,5,7 show a similar behaviour, and should thus be attributed to local ground states. States 4,6 instead have a reduced $r \approx 3 \mu \mathrm{eV}$ and a significant $p \approx 3 \mu \mathrm{eV}$. They are excited states of state 1 , as can be 

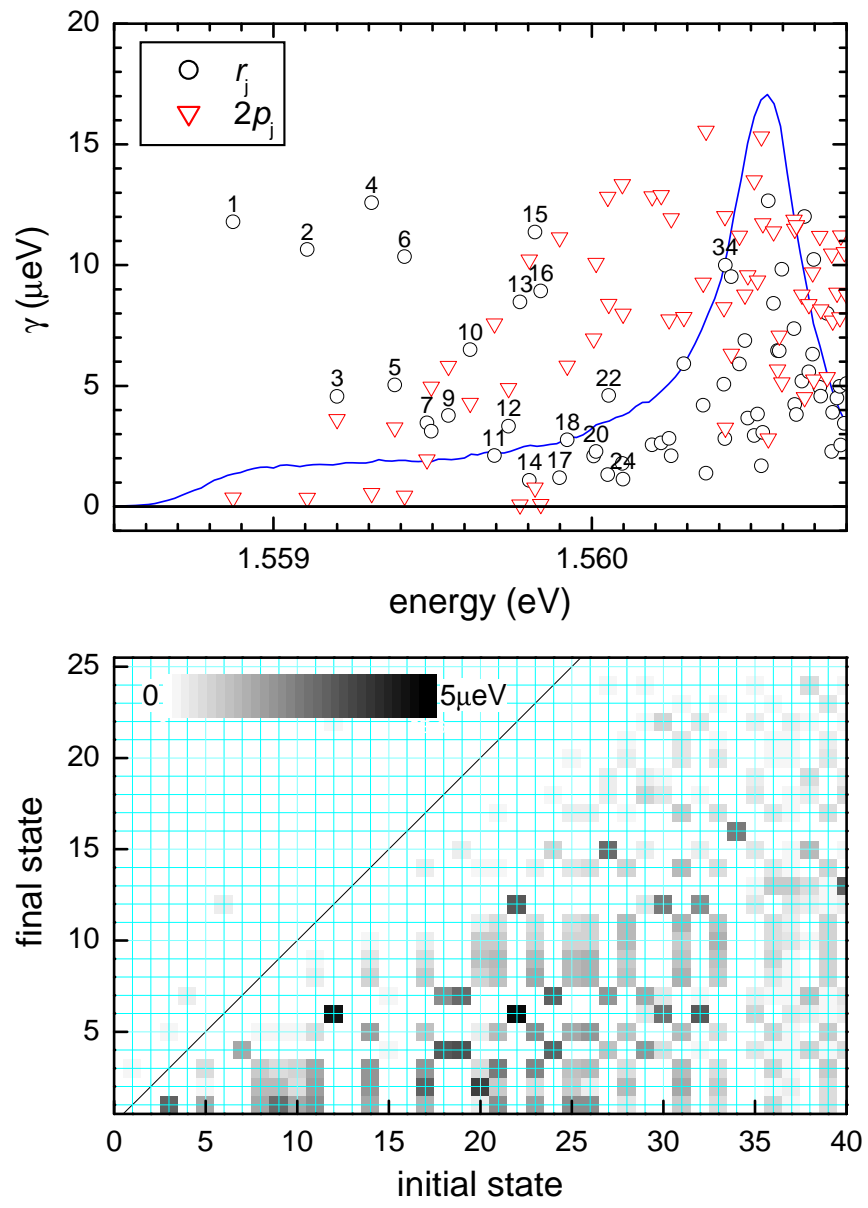

FIG. 18: As Fig.16 for a different disorder potential realization $V_{\mathrm{II}}(\mathbf{R})$.

seen from the phonon scattering matrix $p_{l j}$ given in the lower panel of Fig.16] which shows sizable $p_{14}$ and $p_{16}$, but negligible $p_{24}, p_{34}, p_{26}, p_{36}$. From the $p_{l j}$, we find also that the states $11,14,15$ are further excited states of state 1, showing also relaxation into the states 4,6 . Similarly, states $9,10,18,20,23$ are excited states of 2 , and $8,12,17,25,27$ are excited states of 3 . Energetically close to the $38 \mathrm{ML}$ potential the local ground state 34 is formed, which has an exceptionally large $r \approx 19 \mu \mathrm{eV}$, indicating its large spatial extension.

This discussion can be verified by observing the spatial wavefunctions of the various states, which are displayed in Fig.17 The states 1-4-6 represent a s- $\mathrm{p}_{x}-\mathrm{p}_{y}$ sequence, where the s,p,d,e,.. states refer to the sequence of quantized state groups in a two-dimensional harmonic oscillator which have the degeneracies $1,2,3,4, \ldots$. The higher excited states 11,14 (and 15, not shown) are d-states with 2 nodes, but are already quite deformed due to the nonperfect circular symmetry of the potential trench. Similar sequences are observed for the states 2-9-10-18-20-23, 3-8-12-17-25-27 and 7-16-24-29-38. In the sequence 5-1319-22-31 the asymmetry of the trench is so strong that the $\mathrm{d}$ and e states are strongly mixed. The state 34 is a

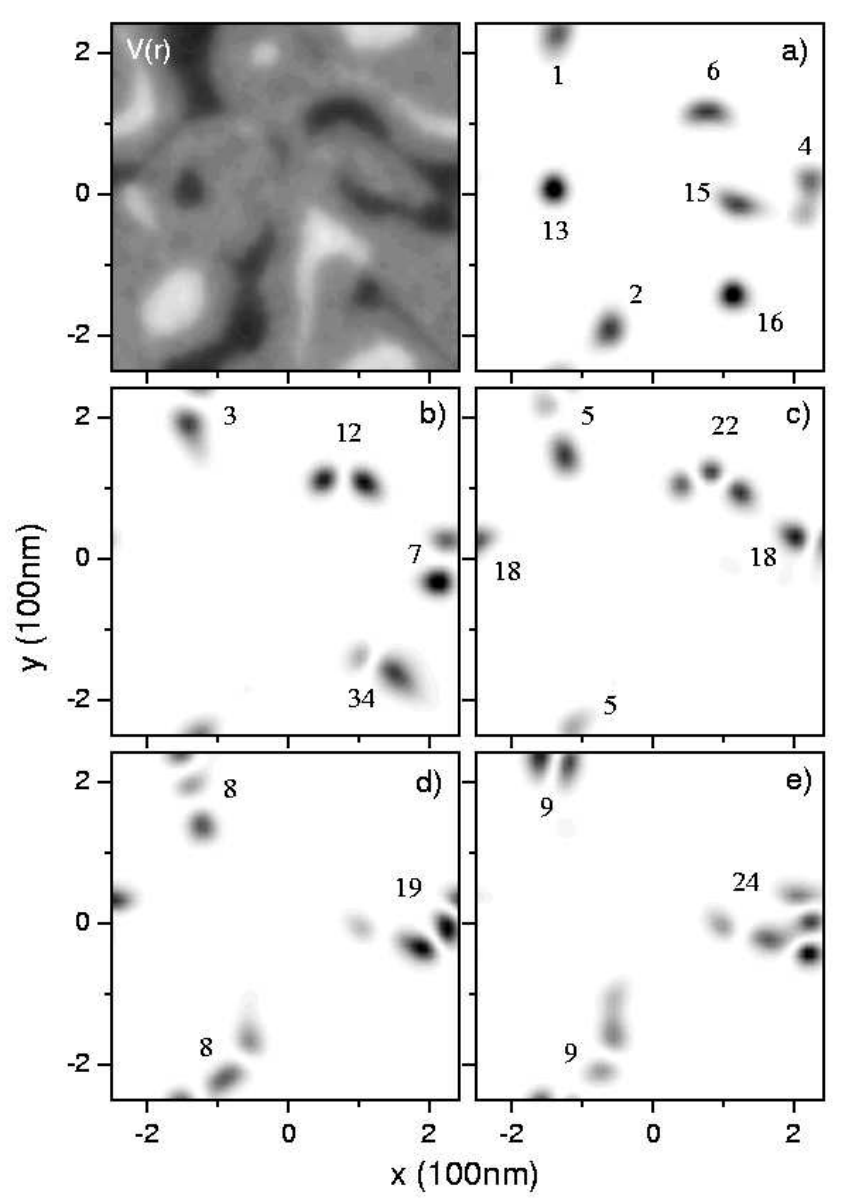

FIG. 19: As Fig.19 for the disorder potential realization $V_{\mathrm{II}}(\mathbf{R})$.

rather large state localized by a $39 \mathrm{ML}$ trench about the size of $a_{\mathrm{B}}$, for which the confinement potential is therefore already reduced due to the averaging over the exciton relative wavefunction $\left|\phi\left(\mathbf{R}^{\prime}\right)\right|^{2}$.

Generally we can say that for realization I, most of the states below the $38 \mathrm{ML}$ peak are well described by ground and excited states of single rather round ML island trenches. In realization II, most of the trenches are instead elongated and bending, some even show bifurcations (see Fig.19). Accordingly, the states localized in the trenches do not resemble the s-p-d state sequence. Looking at $r$ and $p$ in Fig.18 the states 1,2,4,6,13,15,16 are local ground states. State 1 has the excited states 3,5, and shares the excited states $8,9,10,11$ with state 2 . This is due to the connected nature of the trenches, which support multiple local ground states within a trench structure. Such a structure results in a exciton state system with two metastable local ground states (here 1,2), and excited states delocalized over both ground states (here $8,9,10,11$ ) which allow to coherently couple the two local ground states by coulomb and exchange interaction with the excited state. Such coherent coupling has been observed in recent literature using nonlinear 


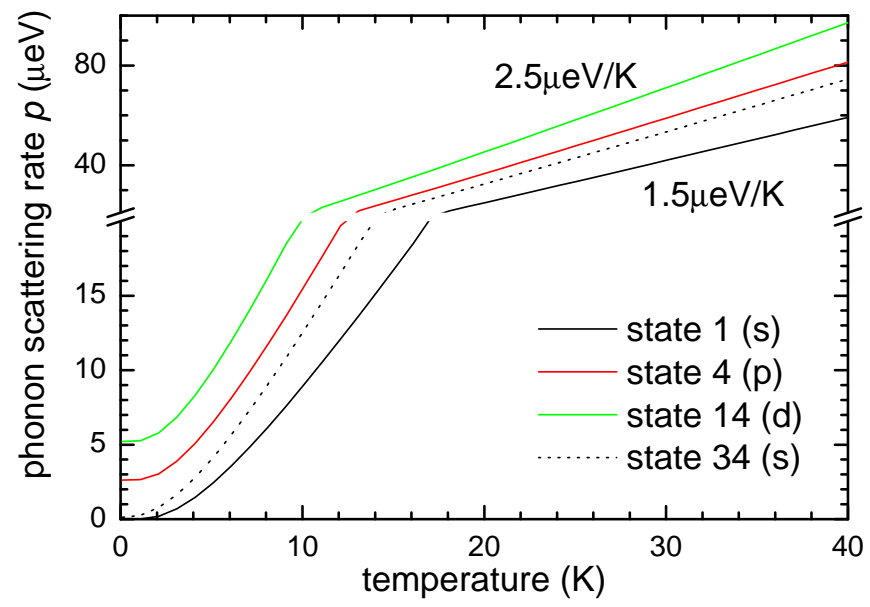

FIG. 20: Temperature dependence of the phonon scattering rate $p_{j}$ for selected exciton states of the disorder potential realization $V_{\mathrm{I}}(\mathbf{R})$.

spectroscopy $\stackrel{47,48}{ }$.

The different localization of the states results not only in different zero temperature dephasing rates, but also in a different temperature dependence. Strongly localized states show an activated temperature dependence. Especially the local ground states (s-like) do show vanishing phonon assisted dephasing at zero temperatures, and an activated behavior for $T>0$ proportional to the phonon occupation at the energy of the transition to the first activated states (p-like). This is exemplified in Fig.20 for the states $1(\mathrm{~s}), 4(\mathrm{p}), 14(\mathrm{~d})$ and $34(\mathrm{~s})$ of the first disorder realization (see Fig.[17). State 1 shows the largest activation energy, given by the distance to its first excited states $(4,6)$ of about $0.6 \mathrm{meV}$. The excited states also show an activation on top of the zero-temperature offset, but with a smaller activation energy as the higher excited states are typically energetically due to the finite barrier height. The weakly localized state 34 shows a weaker activated behavior, similar to what would be expected for an ideal quantum well, where phonon energies between about 0.1-1 meV (for a $11 \mathrm{~nm}$ GaAs QW) can scatter the optically active $k=0$ state to exciton states of higher $k^{49,50}$. These predictions are in agreement with measurements of the temperature-dependent line-widths of single exciton states observed in PL experiments 51 . It is interesting to note that not only the activation energy, but also the linear slope for higher temperatures $(k T>1 \mathrm{meV})$ varies between the states. The localiza- tion has thus an effect on the phonon assisted dephasing even at thermal energies much larger than the localization energies, creating a significant distribution in the exciton dephasing rates at elevated temperatures.

\section{CONCLUSIONS}

In conclusion we have presented a model for the disorder of the heterointerfaces in quantum wells that takes into account several important aspects of the interface formation, like surface diffusion, monolayer island formation, segregation \& interdiffusion, and interface correlation. While not attempting to model the growth process microscopically, the main features observed in structural investigations of the heterointerfaces are considered. The spectrally and time-resolved resonant Rayleigh scattering (RRS) spectra calculated for the interface model are in remarkable agreement with both the time-resolved and spectrally resolved measurements, indicating that the main features of the exciton disorder potential are captured by the model. Calculating the RRS using exciton eigenstates and microscopically calculated dephasing rates due to radiative decay and phonon scattering instead of the often used time-propagation model predicts the observed spectrally dependent homogeneous linewidth and the non-exponential RRS dynamics at long times. The calculated localized exciton states in the monolayer islands including their phonon scattering and their dipole moments are also relevant for comparison with experiments on individual excitonic states performed using high spatial resolution 44.45.46. Our result indicates that the present disorder model contains all the important features of semiconductor heterointerfaces fabricated by growth-interrupted molecular beam epitaxy, and can substantially improve our understanding in a large variety of experimental situations.

\section{Acknowledgments}

The sample was grown by K. Leosson and J.Riis Jensen at the III-V Nanolab, a joint laboratory between Research Center COM and the Niels Bohr Institute, Copenhagen University. The authors thank K. Leosson and G. Kocherscheidt for contributions to the experiments. V. S. acknowledges financial support from the Swiss National Science Foundation, through project No. 620066060 .
1 C. A. Warwick and R. F. Kopf, Appl. Phys. Lett. 60, 386 (1992).

2 D. Bimberg, F. Heinrichsdorff, R. K. Bauer, D. Gerthsen, D. Stenkamp, D. E. Mars, and J. N. Miller, J. Vac. Sci. \& Technol. B 10, 1793 (1992).

${ }^{3}$ R. F. Kopf, E. F. Schubert, T. D. Harris, R. S. Becker, and
G. H. Gilmer, J. Appl. Phys. 74, 6139 (1993).

${ }^{4}$ G. Bernatz, S. Nau, R. Rettig, H. Jänsch, and W. Stolz, J. Appl. Phys. 86, 6752 (1999).

${ }^{5}$ H. Castella and J. W. Wilkins, Phys. Rev. B 58, 16186 (1998).

6 D. Gammon, B. V. Shanabrook, and D. S. Katzer, Phys. 
Rev. Lett. 67, 1547 (1991).

7 V. Savona and R. Zimmermann, Phys. Rev. B 60, 4928 (1999).

8 G. Kocherscheidt, W. Langbein, G. Mannarini, and R. Zimmermann, Phys. Rev. B 66, 161314(R) (2002).

${ }^{9}$ G. Kocherscheidt, W. Langbein, U. Woggon, V. Savona, R. Zimmermann, D. Reuter, and A. D. Wieck, Phys. Rev. B 68, 085207 (2002).

10 W. Langbein, R. Zimmermann, E. Runge, and J. M. Hvam, phys. stat. sol (b) 221, 349 (2000).

11 R. Zimmermann, E. Runge, and V. Savona, in Quantum Coherence, Correlation and Decoherence in Semiconductor Nanostructures, edited by T. Takagahara (Elsevier Science, USA, 2003), p. 89.

12 C. Heyn and M. Harsdorff, Phys. Rev. B 55, 7034 (1997).

${ }^{13}$ K. Leosson, J. R. Jensen, W. Langbein, and J. M. Hvam, Phys. Rev. B 61, 10322 (2000).

14 D. D. Smith, M. Dutta, X. C. Liu, A. F. Terzis, A. Petrou, M. W. Cole, and P. G. Newman, Phys. Rev. B 40, 1407 (1989).

15 I. V. Ponomarev, L. I. Deych, and A. A. Lisyansky, Appl. Phys. Lett. 85, 2496 (2004).

16 W. Langbein, J. M. Hvam, and R. Zimmermann, Phys. Rev. Lett. 82, 1040 (1999).

17 G. Bernatz, S. Nau, R. Rettig, and W. Stolz, IEEE J. Electr. Mat. 29, 129 (2000).

${ }^{18}$ H. Yu, P. B. Mookherjee, R. Murray, and A. Yoshinaga, J. Appl. Phys. 77, 1217 (1995).

19 B. Orschel, G. Oelgart, and R. Houdré, Appl. Phys. Lett. 62, 843 (1993).

${ }^{20}$ U. Jahn, K. Fujiwara, J. Menniger, R. Hey, and H. T. Grahn, J. Appl. Phys. 77, 1211 (1995).

${ }^{21}$ R. F. Kopf, E. F. Schubert, T. D. Harris, and R. S. Becker, Appl. Phys. Lett. 58, 631 (1991).

${ }^{22}$ L. Gottwaldt, K. Pierz, F. J. Ahlers, and E. O. Göbel, J. Appl. Phys. 94, 2464 (2003).

23 D. Gammon, E. S. Snow, B. V. Shanabrook, D. S. Katzer, and D. Park, Phys. Rev. Lett. 76, 3005 (1996).

24 J. R. Jensen, J. M. Hvam, and W. Langbein, J. Appl. Phys. 86, 2584 (1999).

25 E. Runge and R. Zimmermann, Phys. Rev. B 61, 4786 (2000).

26 G. Kocherscheidt, W. Langbein, and V. Savona, phys. stat. sol. (b) 238, 486 (2003).

27 W. Langbein, G. Kocherscheidt, and R. Zimmermann, $O p$ tics of Semiconductors and Their Nanostructures, Vol. 146 of Solid-State Series (Springer, Berlin, 2004), pp. 47-72.

28 G. R. Hayes, B. Deveaud, V. Savona, and S. Haacke, Phys. Rev. B 62, 6952 (2000).

29 D. Birkedal and J. Shah, Phys. Rev. Lett. 81, 2372 (1998).

30 G. Mannarini, R. Zimmermann, G. Kocherscheidt, and W. Langbein, phys. stat. sol. (b) 238, 494 (2003).

31 M. Gurioli, A. Vinattieri, J. Martinez-Pastor, and M.Colocci, Phys. Rev. B 50, 11817 (1994).

32 K. Leósson, Ph.D. thesis, Technical University of Denmark, 2001.

33 M. Gurioli, J. Martinez-Pastor, M. Colocci, A. Bosacchi, S. Franchi, and L. C. Andreani, Phys. Rev. B 47, 15755 (1993).
${ }^{34}$ L. Andreani, in Confined Electrons and Photons, Vol. B 340 of NATO ASI Series, edited by E. Burstein and C. Weisbuch (Plenum Press, New York, 1995), p. 57.

35 K. Siantidis, V. M. Axt, and T. Kuhn, Phys. Rev. B 65, 035303 (2001).

36 M. Grochol, F. Grosse, and R. Zimmermann, Phys. Rev. B 71, 125339 (2005).

37 V. Türck, S. Rodt, O. Stier, R. Heitz, R. Engelhardt, U. W. Pohl, D. Bimberg, and R. Steingrüber, Phys. Rev. B 61, 9944 (2000).

38 J. Erland, J. C. Kim, N. H. Bonadeo, D. G. Steel, D. Gammon, and D. S. Katzer, Phys. Rev. B 60, R8497 (1999).

39 M. Sugawara, Phys. Rev. B 51, 10743 (1995).

40 L. C. Andreani, G. Panzarini, and J.-M. Gérard, Phys. Rev. B 60, 13276 (1999).

41 J. Hours, P. Senellart, E. Peter, A. Cavanna, and J. Bloch, Phys, Rev. B 71, 161306(R) (2005).

42 W. Langbein, phys. stat. sol. (b) 234, 84 (2002).

43 W. Langbein, Speckle-Analysis of Resonant Light Emission from Solids (Habilitationsschrift, Universität Dortmund, Dortmund, 2002).

44 Q. Wu, R. D. Grober, D. Gammon, and D. S. Katzer, Phys. Rev. Lett. 83, 2652 (1999).

45 J. Guest, T. H. Stievater, G. Chen, E. A. Tabak, B. G. Orr, D. G. Steel, D. Gammon, and D. S. Katzer, Science 293, 2224 (2001).

46 W. Langbein and B. Patton, Phys. Rev. Lett. 95, 017403 (2005).

47 E. T. Batteh, J. Cheng, G. Chen, D. G. Steel, D. Gammon, D. S. Katzer, and D. Park, Phys. Rev. B 71, 155327 (2005).

48 T. Unold, K. Mueller, C. Lienau, T. Elsaesser, and A. D. Wieck, Phys. Rev. Lett. 94, 137404 (2005).

49 A. Thränhardt, S. Kuckenberg, A. Knorr, T. Meier, and S. Koch, Phys. Rev. B 62, 2706 (2000).

50 C. Piermarocchi, F. Tassone, V. Savona, A. Quattropani, and P. Schwendimann, Phys. Rev. B 53, 15834 (1996).

51 D. Gammon, E. S. Snow, B. V. Shanabrook, D. S. Katzer, and D. Park, Science 273, 87 (1996).

52 The electron and hole wavefunctions in the in-plane coordinate $\mathbf{R}^{\prime}$ are $\propto \exp \left(-M / m_{e, h}\left|\mathbf{R}-\mathbf{R}^{\prime}\right| / a_{\mathrm{B}}\right)$ with the in-plane masses $m_{e, h}$ of electrons and holes. For $m_{e}=m_{h}$, the relationship used in our work is obtained. In other cases, the convolution function should contain a sum of the electron and hole probability, weighted with the respective interface potential (see Ref.11). Within the rigid exciton approximation, the difference to the chosen approach is not relevant.

53 In this case, our model results in a vanishing disorder potential, as the change in the absolute exciton position in z-direction is not considered. However, realistically such a disorder contribution would exist, giving rise to repulsive potentials at the ML steps. We expect these contributions to the disorder potential to be weak compared to the ML thickness fluctuations as long as their lateral extension is larger than the exciton bohr radius.

54 As the exciton wavefunction is not constant within the well, a weighted averaging should be performed. 